\title{
Large scale Sobolev inequalities on metric measure spaces and applications
}

Romain Tessera

\begin{abstract}
For functions on a metric measure space, we introduce a notion of "gradient at a given scale". This allows us to define Sobolev inequalities at a given scale. We prove that satisfying a Sobolev inequality at a large enough scale is invariant under large-scale equivalence, a metric-measure version of coarse equivalence. We prove that for a Riemmanian manifold satisfying a local Poincaré inequality, our notion of Sobolev inequalities at large scale is equivalent to its classical version. These notions provide a natural and efficient point of view to study the relations between the large time on-diagonal behavior of random walks and the isoperimetry of the space. Specializing our main result to locally compact groups, we obtain that the $L^{p}$-isoperimetric profile, for every $1 \leq p \leq \infty$ is invariant under quasiisometry between amenable unimodular compactly generated locally compact groups. A qualitative application of this new approach is a very general characterization of the existence of a spectral gap on a quasi-transitive measure space $X$, providing a natural point of view to understand this phenomenon.
\end{abstract}

\section{Introduction}

We introduce a notion of "gradient at a certain scale" for bounded functions defined on a general metric measure space. This allows us to define Sobolev inequalities "at a given scale". We say that a Sobolev inequality holds "at large scale" if it holds at scale $h$, with $h$ large enough. The central result of this paper is to show that these so-called large-scale Sobolev inequalities are

2000 Mathematics Subject Classification: 20F65, 22A10.

Keywords: Large-scale analysis on metric spaces, coarse equivalence, symmetric random walks on groups, Sobolev inequalities, isoperimetry. 
invariant under large-scale equivalence (large-scale equivalence is the natural metric-measure version of coarse equivalence). Moreover, we show that under some uniform (discrete) connectivity assumption, large-scale Sobolev inequalities are equivalent to Sobolev inequalities at some explicit scale. We also study the relations between our notion of gradient at given scale and the well-known infinitesimal notion of upper-gradient. We generalize some stability results [10] for Sobolev inequalities from the contexts of Riemannian manifolds and of weighted graphs to our general context. The advantage of our point of view is to get rid of any condition at small scale and to work in "highly non-geodesic" spaces. This latter generality may be really useful, for instance when one has to deal with a subspace of a metric space, which can be quite wild in general. This level of generality is also necessary for the study of (non-necessarily compactly generated) locally compact groups where no nice local structure is available. Moreover, note that a locally compact group has no quasi-geodesic left invariant proper metric unless it is compactly generated (see Proposition 6.6). This functional analysis approach generalizes the purely geometric notion of large-scale isoperimetry that we introduced in [30].

These notions provide a natural and efficient point of view to study the relations between the large time on-diagonal behavior of random walks and the isoperimetry of the space. In particular, we obtain that, under mild assumptions on a metric measure space, upper bounds on the probability of return of symmetric random walks are characterized by large-scale Sobolev inequalities, and therefore are invariant under large-scale equivalence (see Theorem 3.5 for a precise statement).

As a qualitative application, we prove that a reversible random walk on a quasi-transitive measure space has spectral radius equal to 1 if and only if the group acting is amenable and unimodular. This provides a general and direct explanation for a phenomenon that has been pointed out in particular cases $^{1}[20,1,27,29,22,26]$.

Notation: Throughout this paper, $B(x, r)$ will denote the closed ball of center $x$, and of radius $r$. If $A$ is a measurable subset of a measure space $(X, \mu), L^{p}(A)$ will denote the space of functions in $L^{p}(X)$, supported in $A$, for every $1 \leq p \leq \infty$.

\section{Statement of the main results in the homogeneous setting}

Let us present our results in a very special -though interesting-case, namely when $X=G$ is a group. Let $G$ be a locally compact, compactly generated

\footnotetext{
${ }^{1}$ Note that some of the results of these articles are more precise than ours and in a sense, more general when they manage to deal with non-reversible random walks.
} 
group equipped with a left-invariant Haar measure $\mu$. Let $S$ be a symmetric compact neighborhood of 1 in $G$ such that $\bigcup_{n \in \mathbb{N}} S^{n}=G$. Equip $G$ with its left-invariant word metric associated to $S$, i.e. $d_{S}(g, h)=\inf \left\{n, g^{-1} h \in S^{n}\right\}$. In particular, we have $B(1, n)=S^{n}$ for all $n \in N$.

\section{Quantitative results}

Recall that a quasi-isometry between two metric spaces $\left(X, d_{X}\right)$ and $\left(Y, d_{Y}\right)$ is a map $F: X \rightarrow Y$ which is bi-Lipschitz for large distances, i.e.

$$
C^{-1} d_{X}(x, y)-C \leq d_{Y}(F(x), F(y)) \leq C d_{X}(x, y)+C
$$

for any $x, y \in X, C$ being a positive constant; and almost surjective, i.e.

$$
\sup _{z \in Y} d(z, F(X))<\infty
$$

Let $\lambda$ be the action of $G$ by left-translations on functions on $G$, i.e. $\lambda(g) f(x)=f\left(g^{-1} x\right)$. For any $1 \leq p \leq \infty$, and any subset $A$ of $G$, define

$$
J_{p}(A)=\sup _{\left.f \in L^{(} A\right)} \frac{\|f\|_{p}}{\sup _{s \in S}\|f-\lambda(s) f\|_{p}} .
$$

We can define two kinds of " $L^{p}$-isoperimetric profile", depending on if we want to optimize $J_{p}(A)$ fixing the volume of $A$, or its diameter. In the first case, we obtain what is often called the $L^{p}$-isoperimetric profile (see for instance $[6,7])$,

$$
j_{G, p}(v)=\sup _{\mu(A)=v} J_{p}(A) .
$$

In the second case, we obtain what we call the $L^{p}$-isoperimetric profile inside balls since it is given by

$$
J_{G, p}^{b}(n)=J_{p}(B(1, n))
$$

We will be interested in the "asymptotic behavior" of these nondecreasing functions. Precisely, let $f, g: \mathbb{R}_{+} \rightarrow \mathbb{R}_{+}$be nondecreasing functions. We write respectively $f \preceq g, f \prec g$ if there exists $C>0$ such that $f(t)=$ $O(g(C t))$, resp. $f(t)=o(g(C t))$ when $t \rightarrow \infty$. We write $f \approx g$ if both $f \preceq g$ and $g \preceq f$. The asymptotic behavior of $f$ is its class modulo the equivalence relation $\approx$.

Now, we can state our main results in this setting. 
828 R. TESSERA

Theorem 1. (See Corollary 10.2) Assume that $(G, S)$ and $(H, T)$ are two unimodular compactly generated, locally compact groups, equipped with symmetric generating subsets $S$ and $T$ respectively. Then, the asymptotic behaviours of $j_{G, p}, J_{G, p}^{b}$, for any $1 \leq p \leq \infty$ do not depend on $S$. Moreover, if $G$ is quasi-isometric to $H$, then

$$
j_{G, p} \approx j_{H, p}, \quad \text { and } \quad J_{G, p}^{b} \approx J_{H, p}^{b} .
$$




\section{A qualitative result}

We also derive a qualitative result on quasi-transitive spaces. Let $G$ be a locally compact, compactly generated group. Let $(X, \mu)$ be a quasi-transitive $G$-space, i.e. a locally compact Borel measure space on which $G$ acts measurably, co-compactly, properly, and almost preserving the measure $\mu$, i.e.

$$
\sup _{g \in G} \sup _{x \in X} \frac{d(g \cdot \mu)}{d \mu}(x)<\infty \text {. }
$$

For every $x \in X$, let $\nu_{x}$ be a probability measure on $X$ which is absolutely continuous with respect to $\mu$. We assume that there exist $S \subset S^{\prime}$, two compact generating subsets of $G$, and a compact subset $K$ of $X$ satisfying $G K=X$, such that for every $x \in X$, the support of $\nu_{x}$ is contained in $g S^{\prime} K$, for some $g \in G$ such that $x \in g S K$. Let us also suppose that $\left(d \nu_{x} / d \mu\right)(y)$ is larger than a constant $c>0$ for $y$ in $g S K$. Denote by $P$ the Markov operator on $L^{2}(X)$ defined by

$$
P f(x)=\int f(y) d \nu_{x}(y)
$$

We make the (important) assumption that $P$ is self-adjoint.

Theorem 2. (see Theorem 11.4 and Corollary 11.14) The following are equivalent

- the spectral radius of $P$ is less than 1 ;

- $G$ is either non-unimodular or non-amenable.

- $G$ is quasi-isometric to a graph (of bounded degree) with positive Cheeger constant.

This theorem is a slight generalization of the following recent result of Saloff-Coste and Woess [26], which they obtained by completely different (and less elementary) methods.

Corollary 3. [26] Let $(X, d)$ be a geodesic metric space and let $G$ be a closed subgroup of Isom $(M)$ acting co-compactly on $X$ by isometries. Fix $r>0$. Then $G$ is unimodular and amenable if and only if the spectral radius of the average operator on balls of radius $r$ is 1 .

Our approach unifies the following results, enhancing their "large-scale" nature. An obvious particular case is when the space $X$ is the group itself. 
Corollary 4. Let $G$ be a locally compact group equipped with a left Haar measure $\mu$. Then $G$ is unimodular and amenable if and only if for every compactly supported, symmetric (with respect to $\mu$ ) random walk on $G$ has spectral radius 1 .

Corollary 5. [27] Let $X$ be a connected graph of bounded degree and let $G$ be a closed subgroup of $\operatorname{Aut}(X)$ such that $X / \operatorname{Aut}(X)$ is finite. Then $G$ is unimodular and amenable if and only if the spectral radius of the simple random walk equals 1 .

When $G$ is transitive this theorem has been proved in [29].

Corollary 6. [26] (see Corollary 11.6) Let $M$ be a Riemannian ${ }^{2}$ manifold and let $G$ be a closed subgroup of Isom $(M)$ acting co-compactly on $M$. Then $G$ is unimodular and amenable if and only if the spectral radius of the heat kernel on $M$ equals 1 , or in other words, if the (Riemannian) Laplacian on $M$ has no spectral gap at zero.

The case where $G$ is transitive has been treated in [22] and the case where $M$ is the universal cover of a compact manifold has been proved in [1].

\section{Organization of the paper}

- In Section 2, we introduce a notion of Sobolev inequalities that captures the geometry at a scale larger than $h>0$.

- In Section 3, we discuss the geometric and probabilistic interpretations of these Sobolev inequalities.

- In Section 4, we discuss the relations between Sobolev inequalities and the isoperimetric profile.

- In Section 5, we introduce the notion of large-scale equivalence, which is a metric-measure version of the well-known notion of coarse equivalence (see [24]).

- In Section 6, we discuss some examples of large-scale equivalences in the contexts of locally compact groups, manifolds, graphs etc.

- In Section 7, we prove a technical but important fact: the definition of large-scale Sobolev inequalities does not depend on the choice of a "large-scale" gradient.

\footnotetext{
${ }^{2}$ Actually the authors give a method that allows them to treat a large class of examples, like all the examples given here, included the case (not explicitly mentioned) of a locally compact, compactly generated group.
} 
- In Section 8.3, we prove our main result, namely that large-scale Sobolev inequalities are invariant under large-scale equivalence.

- In Section 9, we relate Sobolev inequalities with upper bounds on the probability of return of symmetric random walks.

- In Section 10, we discuss the validity of Sobolev inequalities at a given scale when it is true at large-scale. In particular, in Sections 10.2 and 10.3, we prove that under some mild local assumptions, the largescale Sobolev inequalities are equivalent to their classical versions on a Riemaniann manifold.

- Finally, in Section 11, we prove the results announced in the introduction in the context of locally compact groups and quasi-transitive spaces.

\section{Functional analysis at a given scale}

\subsection{Local norm of gradient at scale $h$}

Let $(X, d)$ be a metric space. The purpose of this section is, given a function $f: X \rightarrow \mathbb{R}$, to introduce a quantity that measures the variations of $f$ at a certain scale - say $h$. This notion will have to play the same role as for instance the length of the gradient $|\nabla f(x)|$ on a Riemannian manifold, or its well-known version for graphs (see [6] for instance). For this reason, we will call it a "local norm" of the gradient of $f$, even if no real notion of gradient is introduced (see Remark 2.6 for an alternative approach).

The first naive idea to do this is to define

$$
|\nabla f|_{h}(x)=\sup _{y \in B(x, h)}|f(y)-f(x)|
$$

for any function $f \in L^{\infty}(X), B(x, h)$ denoting the closed ball of center $x$ and radius $h$. Note that this can be written in the following form:

$$
|\nabla f|_{h}(x)=\|f-f(x)\|_{\infty, B(x, h)}
$$

which emphasizes the fact that we actually consider a "local" $L^{\infty}$-norm. Naturally, we would like to define also the local $L^{p}$-norm of the gradient of $f$, for every $1 \leq p \leq \infty$. For this, we obviously need a measure on $X$. What we could do is start from a measure on $X$ and define a local $L^{p}$-norm as the $L^{p}$ norm restricted to balls with respect to this measure. However, when we consider a random process on $X$, the notion of local $L^{2}$-norm that naturally emerges is the $L^{2}$-norm with respect to the probability transition. This motivates the following definition. 
Let $(X, d, \mu)$ be a metric measure space. Consider a family $P=\left(P_{x}\right)_{x \in X}$ of probability measures on $X$. Then for every $p \in[1, \infty]$, we define an operator $|\nabla|_{P, p}$ on $L^{\infty}(X)$ by

$\forall f \in L^{\infty}(X), \quad|\nabla f|_{P, p}(x)=\|f-f(x)\|_{P_{x}, p}=\left(\int|f(y)-f(x)|^{p} d P_{x}(y)\right)^{1 / p}$, if $p<\infty$; and for $p=\infty$, we decide that

$$
|\nabla f|_{P, \infty}(x)=\|f-f(x)\|_{P_{x}, \infty}=\sup \left\{|f(y)-f(x)|, y \in \operatorname{Supp}\left(P_{x}\right)\right\} .
$$

Definition 2.1. A family of probabilities $P=\left(P_{x}\right)_{x \in X}$ on $X$ is called a viewpoint at scale $h>0$ on $X$ if there exist a large constant $1 \leq A<\infty$ and a small constant $c>0$ such that for ( $\mu$-almost) every $x \in X$ :

- $P_{x} \ll \mu$;

- $p_{x}=d P_{x} / d \mu$ is supported in $B(x, A h)$;

- $p_{x}$ is larger than $c$ on $B(x, h)$.

Remark 2.2. Note that a viewpoint at scale $h$ is also a viewpoint at scale $h^{\prime}$ for any $h^{\prime}<h$.

Example 2.3. A basic example of viewpoint at scale $h$ is given by

$$
P_{x}=\frac{1}{V(x, h)} 1_{B(x, h)}, \quad \forall x \in X,
$$

where $V(x, r)$ denotes the volume of the closed ball centered at $x$ of radius $r$. We denote the associated $L^{p}$-gradient by $|\nabla|_{h, p}$. Note that with our previous notation,

$$
|\nabla|_{h}=|\nabla|_{h, \infty}
$$

Example 2.4. (For more details, see [6]) To any connected simplicial graph, we associate a metric measure space, whose elements are the vertices of the graph, the measure is the counting measure and the distance is the usual discrete geodesic distance for which two distinct points joined by an edge are at distance 1 from one another. For simplicity, we will simply call such a metric measure space a graph. The usual discrete local norm of gradient on a graph, usually denoted by $|\nabla f(x)|$, corresponds ${ }^{3}$ with our notations to

$$
|\nabla f|_{1,2}(x)=\left(\frac{1}{V(x, 1)} \sum_{y \in B(x, 1)}|f(x)-f(y)|^{2}\right)^{1 / 2} .
$$

\footnotetext{
${ }^{3}$ In [6], they consider a slightly different definition, where the average is taken over the set of neighbors of $x$ instead of the ball $B(x, 1)$.
} 
Remark 2.5. (Interpretations of the notion of viewpoint at scale $\boldsymbol{h}$ ). A viewpoint at scale $h$ has at least two interesting interpretations: one as a transition operator of a random walk on $X$; the other as a Markov operator acting on $L^{p}(X)$ for every $p \geq 1$. This operator is defined by

$$
\operatorname{Pf}(x)=\int_{X} f(y) d P_{x}(y)
$$

Consequently, there is a natural semi-group structure on the set of viewpoints at scale $h$ on space $X$. Indeed, it is straightforward to check ${ }^{4}$ that if $P$ is a viewpoint at scale $h$ and $Q$ is a viewpoint at scale $h^{\prime}$, then $P \circ Q$ is a viewpoint at any scale $h "$, with $h^{\prime \prime} \geq \max \left\{h, h^{\prime}\right\}$. Moreover, if $(X, d)$ is coarsely $b$-geodesic with $b \leq \min \left\{h, h^{\prime}\right\}$ (see Definitions 6.1 ), then $P \circ Q$ is a viewpoint at scale $h "$ for every $h "<h+h^{\prime}$.

Remark 2.6. (Alternative definition of gradient at scale $\boldsymbol{h}$ ). Let us indicate another way of describing the objects that we introduced. Instead of directly defining a local norm of the gradient at scale $h$, we could first define a gradient at scale $h$ on a fiber space over $X$ and then take a local norm of the gradient on the fibers. Here the fiber space would be $Y_{h}=$ $\left\{(x, y) \in X^{2}, d(x, y) \leq h\right\}$ with projection $\pi: Y \rightarrow X$ on the first factor, so that $\pi^{-1}(x)=B(x, h)$. The gradient at scale $h$ of $f$ is then $\nabla_{h} f(x, y)=$ $f(x)-f(y)$, where $(x, y) \in Y_{h}$. A viewpoint at scale $h$ on $X$ is now a probability measure on every fiber of some $Y_{A h}$ for $A$ large enough; and the $L^{p}$-gradient of $f$ associated to such a viewpoint corresponds to the $L^{p}$-norm of $f$ in every fiber with respect to this measure ${ }^{5}$.

\subsection{Laplacian at scale $h$}

We can also define a Laplacian w.r.t. a viewpoint $P=\left(P_{x}\right)_{x \in X}$ by

$$
\Delta_{P} f(x)=(i d-P) f(x)=\int(f(x)-f(y)) d P_{x}(y)
$$

and more generally a $p$-Laplacian for any $p>1$ by

$$
\Delta_{P, p} f(x)=\int|f(x)-f(y)|^{p-2}(f(x)-f(y)) d P_{x}(y
$$

\footnotetext{
${ }^{4}$ One has to suppose that the space is locally doubling, see Definitions 5.1.

${ }^{5}$ Note that we can also define the gradient of $f$ without referring to the scale: $\nabla f$ : $X \times X \rightarrow \mathbb{R}, \nabla f(x, y)=f(x)-f(y)$, looking at $X \times X$ as a fiber space over the first factor. Then the scale appears when choosing a norm on every fiber.
} 
If $P$ is self-adjoint with respect to the scalar product associated to $\mu$, then we have the usual relations

$$
\begin{aligned}
& \left\langle\Delta_{P, p} f, g\right\rangle=\int\left(\int|f(x)-f(y)|^{p-2}(f(x)-f(y))(g(x)-g(y)) d P_{x}(y)\right) d \mu(x), \\
& \left\langle\Delta_{P, p} f, f\right\rangle=\iint|f(x)-f(y)|^{p} p_{x}(y) d \mu(y) d \mu(x)=\left\||\nabla f|_{P, p}\right\|_{p}^{p},
\end{aligned}
$$

and in particular, for $p=2$,

$$
\left\langle\Delta_{P} f, f\right\rangle=\iint|f(x)-f(y)|^{2} p_{x}(y) d \mu(x) d \mu(y)=\left\||\nabla f|_{P, 2}\right\|_{2}^{2} .
$$

In particular, if $A$ is a measurable subset of $X$ with finite volume, The first non-zero eigenvalue $\delta_{P}$ of $\Delta_{P}$ acting $L^{p}(A)$ is

$$
\delta_{P}(A)=\inf _{f \in L^{p}(A)} \frac{\left\||\nabla f|_{P, 2}\right\|_{2}^{2}}{\|f\|_{2}^{2}} .
$$

\subsection{Sobolev inequalities at scale $h$}

Let $\varphi: \mathbb{R}_{+} \rightarrow \mathbb{R}_{+}$be an increasing function and let $p \in[1, \infty]$. The following formulation of Sobolev inequality was first introduced in [5]. We refer to [7] for the link with more classical formulations, for instance in $\mathbb{R}^{n}$.

Definition 2.7. One says that $X$ satisfies a Sobolev inequality $\left(S_{\varphi}^{p}\right)$ at scale (at least) $h>0$ if there exists some finite positive constants $C, C^{\prime}$ depending only on $h, p$ and $\varphi$ such that

$$
\|f\|_{p} \leq C \varphi\left(C^{\prime}|\Omega|\right)\left\||\nabla f|_{h}\right\|_{p}
$$

where $\Omega$ ranges over all compact subsets of $X,|\Omega|$ denotes the measure $\mu(\Omega)$, and $f \in L^{\infty}(\Omega)$.

Definition 2.8. We say that $X$ satisfies a large-scale Sobolev inequality $\left(S_{\varphi}^{p}\right)$ if it satisfies $\left(S_{\varphi}^{p}\right)$ at some scale $h$ (equivalently, for $h$ large enough).

Crucial remark 2.9. Note that to define the Sobolev inequalities at large scale, we arbitrarily chose to write them with $|\nabla|_{h}$ whereas we could have defined them with $|\nabla|_{P, q}$ for any viewpoint $\left(P_{x}\right)_{x \in X}$ at scale $h$ and any $q \geq 1$. A crucial and useful fact that we prove in Section 7 is that satisfying a largescale Sobolev inequality does not depend on this choice.

Remark 2.10. Note that for large-scale Sobolev inequalities, we only need to consider subsets $\Omega$ with large. In fact, we will only be interested in the asymptotic behavior of $\varphi$. 
Remark 2.11. It is easy to prove that $\left(S_{\varphi}^{p}\right)$ implies $\left(S_{\varphi}^{q}\right)$ whenever $p \leq$ $q<\infty$ for any choice of gradient (see [7] for a proof in the Riemannian setting). It is proved in [9] that the converse is false for general Riemannian manifolds. This is likely to be true for groups, although it is still open.

\subsection{Link with Sobolev inequalities for infinitesimal gradients}

Other notions of "local norm of gradient" have been introduced and studied for general metric spaces. In particular the notion of upper gradient plays a crucial role in the study of doubling metric spaces equipped with the Hausdorff measure (see for instance [17, 28], or Definition 10.4). These spaces naturally appear as boundaries of Gromov-hyperbolic spaces and are often studied up to quasi-conformal maps. Such a point of view is quite different from ours since it focuses on the local properties of the space, which is often supposed compact. However, it is natural to ask under what condition on the space a large-scale Sobolev inequality is equivalent to the same Sobolev inequality w.r.t. some upper gradient. In particular, given a Riemannian manifold, is it true that it satisfies a Sobolev inequality at large scale if and only if it satisfies it for its usual gradient? Proposition 10.9 says that if a Riemannian manifold satisfies a Sobolev inequality for its usual gradient, then it also satisfies it at large scale (but the proof is not as obvious as one could expect). However, the converse can be false, for instance if the Riemannian manifold contains a sequence of open submanifolds isometric to open half-spheres of radius going to zero. A sufficient condition to get a positive answer is to ask for a local Poincaré inequality (see Proposition 10.7).

Other ideas for ignoring the local geometry of a Riemannian manifold

Different strategies have been used to ignore the local geometric properties of a manifold. In [3] for instance, they avoid the local behavior of the isoperimetric profile on a manifold by restricting it to subsets containing a geodesic ball of fixed radius. In [4], they consider Nash inequalities restricted to functions convoluted by the heat kernel at time 1 and obtain in this way the invariance under quasi-isometries of certain upper bounds of the on-diagonal behaviour of the heat kernel: this idea is quite closed to ours (see Remark 10.11). This issues are discussed in Sections 10.3 and 10.2. Among other things, we prove under a very weak property of bounded geometry that a manifold satisfies a Sobolev inequality at large scale if and only if it satisfies it for the usual gradient in restriction to functions of the form $g=P f$, where $P$ is the Markov operator associated to any viewpoint at some scale $h>0$. 


\section{Sobolev inequalities $\left(S_{\varphi}^{p}\right)$ at scale $h$ for $p=1,2, \infty$}

Now let us give characterizations of $\left(S_{\varphi}^{p}\right)$ at given scales for the important values of $p=1,2, \infty$ (see [6] for the case of graphs and [7] for Riemannian manifolds).

\subsection{Geometric interpretations of $\left(S_{\varphi}^{p}\right)$ at scale $h$ for $p=1, \infty$}

In [4] (see also [7, proposition 22]), it is proved that $\left(S_{\varphi}^{\infty}\right)$ can only hold if $\varphi$ is unbounded and then is equivalent to the volume lower bound

$$
V(x, r) \geq \varphi^{-1}(r)
$$

where $\varphi^{-1}(r)=\{v, \varphi(v) \geq r\}$, for every $x \in X$ and every $r>0$. The original proof works formally in our setting.

Proposition 3.1. Let $(X, d \mu)$ be a metric measure space. The Sobolev inequality $\left(S_{\varphi}^{\infty}\right)$ at scale $h$ can only hold if $\varphi$ is unbounded and then is equivalent to the volume lower bound

$$
V(x, r) \geq \varphi^{-1}(r)
$$

for $r \geq h$.

The inequality $\left(S_{\varphi}^{1}\right)$ at scale $h$ is equivalent to the isoperimetric inequality (at scale $h$ )

$$
\frac{\left|\partial_{h} \Omega\right|}{|\Omega|} \geq \frac{1}{C \varphi\left(C^{\prime}|\Omega|\right)}
$$

where the boundary of $A$ is defined by

$$
\partial_{h} A=[A]_{h} \cap\left[A^{c}\right]_{h}
$$

with the usual notation $[A]_{h}=\{x \in X, d(x, A) \leq h\}$. The usual proof of this equivalence (see [7]) works formally in our context, using the following version of the co-area formula:

$$
\frac{1}{2} \int_{\mathbb{R}_{+}} \mu\left(\partial_{h}\{f \geq t\}\right) d t \leq \int_{X}|\nabla f|_{h}(x) d \mu(x) \leq \int_{\mathbb{R}_{+}} \mu\left(\partial_{h}\{f \geq t\}\right) d t
$$

where $f$ is a non-negative measurable function defined on $X$. Indeed, for every measurable subset $A \subset X$, we have

$$
\mu\left(\partial_{h} A\right)=\int_{X}\left|\nabla 1_{A}\right|_{h}(x) d \mu(x) .
$$


Thus, (3.1) follows by integrating over $X$ the following local inequalities

$$
\frac{1}{2} \int_{\mathbb{R}_{+}}\left|\nabla 1_{\{f \geq t\}}\right|_{h}(x) d t \leq|\nabla f|_{h}(x) \leq \int_{\mathbb{R}_{+}}\left|\nabla 1_{\{f \geq t\}}\right|_{h}(x) d t,
$$

for every $x \in X$. The right-hand inequality results from the fact that $f=$ $\int_{\mathbb{R}_{+}} 1_{\{f \geq t\}} d t$ and from the sub-additivity of $|\nabla|_{h}$. To prove the left-hand, note that $\left|\nabla 1_{\{f \geq t\}}(x)\right|_{h}=1$ if and only if

$$
\inf _{B(x, h)} f<t \leq \sup _{B(x, h)} f
$$

or

$$
\inf _{B(x, h)} f \leq t<\sup _{B(x, h)} f
$$

Hence,

$$
\int_{\mathbb{R}_{+}}\left|\nabla 1_{\{f \geq t\}}\right|_{h}(x) d t \leq \sup _{B(x, h)} f-\inf _{B(x, h)} f \leq 2|\nabla f|_{h}(x),
$$

which proves (3.2).

\subsection{Probabilistic interpretation of $\left(S_{\varphi}^{2}\right)$ at scale $h$}

The case $p=2$ is of particular interest since it contains some probabilistic information on the space $X$. It is proved in [8] that for manifolds with bounded geometry, upper bounds of the large-time on-diagonal behavior of the heat kernel are equivalent to some Sobolev inequality $\left(S_{\varphi}^{2}\right)$. In [6], a similar statement is proved for the standard random walk on a weighted graph. In Section 9, we give a discrete-time version of this theorem in our general setting. The proof of Theorem 3.5 below emphasizes the fact that the notion of viewpoint at scale $h$ is likely to be the most natural way of capturing the link between large-scale geometry and the long-time behavior of random walks on $X$.

Definition 3.2. Let $(X, d, \mu)$ be a metric measure space and consider some $h>0$. A view-point $P=\left(P_{x}\right)_{x \in X}$ at scale $h$ on $X$ is called symmetric if one of the following equivalent statement holds.

- The random walk whose probability of transition at every $x \in X$ is given by $P_{x}$ is reversible with respect to the measure $\mu$.

- The associated operator on $L^{2}(X, \mu)$ defined by

$$
P f(x)=\int_{X} f(y) d P_{x}(y)
$$

is self-adjoint.

- For a.e. $x, y \in X, p_{x}(y)=p_{y}(x)$. 
Definition 3.3. We call a reversible random walk at scale $h$ a random walk whose associated transition operator $P$ is a symmetric view-point at scale $h$.

Example 3.4. Let $(X, d, \mu)$ be a metric measure space. Consider the standard viewpoint at scale $h$ of density $p_{x}=1_{B(x, h)} / V(x, h)$ with respect to $\mu$. In general, this is not a symmetric viewpoint, i.e. the random walk of probability transition $d P_{x}(y)=p_{x}(y) d \mu(y)$ is not reversible with respect to $\mu$. However, it is reversible with respect to the measure $\mu^{\prime}$ defined by

$$
d \mu^{\prime}(x)=V(x, h) d \mu(x)
$$

It is easy to check that if $(X, d, \mu)$ is locally doubling, then so is $\left(X, d, \mu^{\prime}\right)$. Moreover, if $x \mapsto V(x, h)$ is bounded from above and from below, then $P$ defines a symmetric viewpoint on $\left(X, d, \mu^{\prime}\right)$.

The relations between large-scale Sobolev inequalities $\left(S_{\varphi}^{2}\right)$ and random walks on a metric measure space are summarized in the following theorem, whose proof is adapted from [7, Theorem 7.2]. We use the notation $d P_{x}^{n}(y)=$ $p_{x}^{n}(y) d \mu(y)$.

Theorem 3.5. (see Section 9) Let $X=(X, d, \mu)$ be a metric measure space and let $P=\left(P_{x}\right)_{x \in X}$ be a symmetric view-point at scale $h$ on $X$. Let $\varphi$ be some increasing positive function. Define $\gamma$ by

$$
t=\int_{0}^{1 / \gamma(t)}(\varphi(v))^{2} \frac{d v}{v} .
$$

(i) Assume that $X$ satisfies a large-scale Sobolev inequality $\left(S_{\varphi}^{2}\right)$. Then

$$
p_{x}^{2 n}(x) \leq \gamma(c n) \quad \forall n \in \mathbb{N}, a . e \forall x \in X
$$

for some constant $c>0$.

(ii) If the logarithmic derivative of $\gamma$ has at most polynomial growth ${ }^{6}$ and if

$$
p_{x}^{2 n}(x) \leq \gamma(n) \quad \forall n \in \mathbb{N}, a . e \forall x \in X,
$$

then $X$ satisfies $\left(S_{\varphi}^{2}\right)$ w.r.t. $|\nabla|_{P, 2}$.

\footnotetext{
${ }^{6}$ This condition, called $(\delta)$ in $[6$, p. 18$]$ is very weak since it is satisfied by all functions $(\log t)^{a} t^{b} e^{c t^{d}}$ for any real numbers $a, b, c, d$.
} 


\section{Sobolev and isoperimetry at scale $h$}

\subsection{Isoperimetric profile at scale $h$}

Generalizing the case $p=1$, Sobolev inequalities $\left(S_{\varphi}^{p}\right)$ can be also understood as $L^{p}$-isoperimetric inequalities. Let $A$ be a measurable subset of $X$. For every $p>0$, define

$$
J_{p}(A)=\sup _{f \in L^{\infty}(A)} \frac{\|f\|_{p}}{\left\||\nabla f|_{h}\right\|_{p}} .
$$

Note that for $p=2$, this is just the square root of the inverse of the first eigenvalue of the Laplacian $\Delta_{P}$ acting on $L^{2}(A)$ (see Section 2.2).

Now, taking the supremum over subsets $A$ with measure less than $m>0$, we get an increasing function $j_{X, p}$ sometimes called the $L^{p}$-isoperimetric profile. Note that the terminology "isoperimetric profile" is somewhat ambiguous since there exist various nonequivalent definitions (see in particular [10, Chapter 1]). One of them is

$$
j_{X}(m)=\sup _{|A| \leq m} \frac{|A|}{\left|\partial_{h} A\right|}
$$

which satisfies

$$
j_{X} \approx j_{X, 1}
$$

taking the same $h$ in the definition of the gradient and in the definition of the boundary. Clearly, the space $X$ always satisfies the Sobolev inequality $\left(S_{\varphi}^{p}\right)$ with $\varphi=j_{X, p}$. Conversely, if $X$ satisfies $\left(S_{\varphi}^{p}\right)$ for a function $\varphi$, then

$$
j_{X, p} \succeq \varphi .
$$

It is easy to check that

$$
j_{X, p} \preceq j_{X, q}
$$

whenever $p \leq q<\infty$ (see Remark 2.11 about Sobolev inequalities).

\subsection{Isoperimetric profile inside balls}

Definition 4.1. Let us fix a gradient at scale $h$ on $X$. The $L^{p}$-isoperimetric profile inside balls is the nondecreasing function $J_{G, p}^{b}$ defined by

$$
J_{X, p}^{b}(t)=\sup _{x \in X} J_{p}(B(x, t))
$$

Note that $J_{X, p}^{b}(t)$ is asymptotically equivalent to the supremum of $J_{p}(A)$ over subsets $A$ of diameter ${ }^{7}$ less than $t$. The $L^{p}$-isoperimetric profile inside

\footnotetext{
${ }^{7}$ This profile is associated to another kind of Sobolev inequalities, where the function $\varphi$ of the volume is replaced by a function $\Phi$ of the diameter.
} 
balls plays a crucial role in the study of uniform embeddings of amenable groups into $L^{p}$-spaces (see [31]). It is also central in the proof [32] that a closed at infinity, homogenous manifold does not carry any non-constant $p$-harmonic function with gradient in $L^{p}$.

\subsection{Link with the large-scale isoperimetry introduced in [30]}

One can also define another kind of isoperimetric profile at scale $h$ :

$$
I(t)=\inf _{\mu(A) \geq t} \mu\left(\partial_{h} A\right)
$$

which can be specialized on a family of (measurable) subsets of finite volume $\mathcal{A}$ : we call lower (resp. upper) profile at scale $h$ restricted to $\mathcal{A}$ the nondecreasing function $I_{\mathcal{A}}^{\downarrow}$ defined by

$$
I_{\mathcal{A}}^{\downarrow}(t)=\inf _{\mu(A) \geq t, A \in \mathcal{A}} \mu\left(\partial_{h} A\right)
$$

(resp. $\left.I_{\mathcal{A}}^{\uparrow}(t)=\sup _{\mu(A) \leq t, A \in \mathcal{A}} \mu\left(\partial_{h} A\right)\right)$. We can then study the large-scale isoperimetric properties of a family $\mathcal{A}$ considering the asymptotic behavior of these two increasing functions [30]. In [30], we used this variant to investigate the question: are balls always asymptotically isoperimetric in a metric measure space with doubling property? For that purpose, we introduced a general setting adapted to the study of asymptotic isoperimetry on metric measure spaces. An important consequence of the geometric interpretation of Sobolev inequalities in $L^{1}$ (see Section 3.1) is that every geometric notion that we introduced in [30, Section 3] appears as a particular case of the functional point of view adopted in the present paper. In particular, [30, Theorem 3.10] that implied the invariance under large-scale equivalence of isoperimetric properties is now covered by the lemmas of Section 8.3. Moreover, we choose here to treat separately the large-scale setting, where no connectivity hypotheses are required on the spaces, and the control on the scale that really depends on a connectivity assumption (see Section 10).

\section{Large-scale Sobolev inequalities}

In this section, we define an equivalence relation, called large-scale equivalence between metric measure spaces. This notion is simply a metric measure version of the notion of coarse equivalence for metric spaces introduced by Roe in [24].

The metric measure spaces that we will consider satisfy a very weak property of bounded geometry introduced in [10]. 
Definition 5.1. We say ${ }^{8}$ that a space $X$ is locally doubling at scale $r>0$ if there exists a constant $C_{r}$ such that

$$
\forall x \in X, \quad V(x, 2 r) \leq C_{r} V(x, r)
$$

where $V(x, r)=\mu(B(x, r))$. If it is locally doubling at every scale $r>0$, then we just say that $X$ is locally doubling.

Crucial remark 5.2. Since the constant $C_{r}$ depends on $r$, the locally doubling property has absolutely no influence on the volume growth. In particular, one should be careful to distinguish it from the well-known doubling property stating that there exists a constant $C<\infty$ (not depending on the radius) such that $V(x, 2 r) \leq C V(x, r)$ for all $x \in X$ and $r>0$. Contrary to the locally doubling property, the doubling property implies polynomial growth, i.e. that there exists a constant $D<\infty$ such that $V(x, r) \leq r^{D} V(x, 1)$ for every $x \in X$ and $r \geq 1$.

For most of the results proved in this paper $^{9}$, we only use the locally doubling property at scale $r \geq h / 2$, if the gradient considered is at scale $h$. However, to simplify the exposition, we will always assume that the space is locally doubling.

Clearly, the locally doubling property is a very weak property of controlled geometry: for instance, every graph with bounded degree, equipped with the counting measure is locally doubling. Other examples are Riemannian manifolds with Ricci curvature bounded from below. Assume that the volume of balls of fixed radius is bounded from above and from below by constants depending on $r$. Then one can check easily that $X$ is locally doubling. It is important to note that the locally doubling property is strictly weaker than this property. One can easily construct weighted graphs or Riemannian manifolds which are locally doubling but with unbounded volume of balls of radius 1 .

Example 5.3. Let $X$ be a connected graph with degree bounded by $d$, equipped with the counting measure. The volume of balls of radius $r$ satisfies

$$
\forall x \in X, \quad 1 \leq V(x, r) \leq d^{r} .
$$

In particular, $X$ is locally doubling.

Example 5.4. Let $(X, d, \mu)$ be a metric measure space and let $G$ be a locally compact group acting by isometries that preserve the measure. If $G$ acts co-compactly, then $X$ is locally doubling.

\footnotetext{
${ }^{8}$ In [10] and in [30], the local doubling property is denoted $(D V)_{l o c}$.

${ }^{9}$ In fact all the results except the few ones where the infinitesimal structure of the space is clearly involved.
} 
Definition 5.5. Let $(X, d, \mu)$ and $\left(X^{\prime}, d^{\prime}, \mu\right)$ two spaces satisfying the locally doubling property. Let us say that $X$ and $X^{\prime}$ are large-scale equivalent if there is a function $F$ from $X$ to $X^{\prime}$ with the following properties

(a) for every sequence of pairs $\left(x_{n}, y_{n}\right) \in\left(X^{2}\right)^{\mathbb{N}}$

$$
\left(d\left(F\left(x_{n}\right), F\left(y_{n}\right)\right) \rightarrow \infty\right) \Leftrightarrow\left(d\left(x_{n}, y_{n}\right) \rightarrow \infty\right) .
$$

(b) $F$ is almost onto, i.e. there exists a constant $C$ such that $[F(X)]_{C}=X^{\prime}$.

(c) For $r>0$ large enough, there is a constant $C_{r}>0$ such that for all $x \in X$

$$
C_{r}^{-1} V(x, r) \leq V(F(x), r) \leq C_{r} V(x, r) .
$$

Crucial remark 5.6. Note that being large-scale equivalent is an equivalence relation between metric measure spaces with locally doubling property.

Remark 5.7. If $X$ and $X^{\prime}$ are quasi-geodesic, then (a) and (b) imply that $F$ is roughly bi-Lipschitz: there exists $C \geq 1$ such that

$$
C^{-1} d(x, y)-C \leq d(F(x), F(y)) \leq C d(x, y)+C .
$$

This is very easy and left to the reader. In this case, (a) and (b) correspond to the classical definition of a quasi-isometry.

Example 5.8. Consider the subclass of metric measure spaces including graphs with bounded degree, equipped with the countable measure; Riemannian manifolds with Ricci curvature bounded from below and sectional curvature bounded from above, equipped with the Riemannian measure. In this class, quasi-isometries are always large-scale equivalences.

\section{Examples}

\subsection{Discretization}

Recall that a weighted graph is a connected graph $X$ equipped with a structure of metric measure space on the set of its vertices, the distance being the usual geodesic one. Similarly, a weighted manifold is a Riemannian manifold equipped with a measure $d \mu$ absolutely continuous with respect to the Riemannian measure. A discretization [14, 19] of a weighted Riemannian manifold $X$ can be defined as a weighted graph large-scale equivalent to $X$. More generally, a discretization of a metric measure space is a weighted graph large-scale equivalent to $X$. 
Consider some $b>0$. We call a $b$-chain between two points $x, y \in X$ a chain $x=x_{1} \ldots x_{m}=y$ such that for every $1 \leq i<m, d\left(x_{i}, x_{i+1}\right) \leq b$. Define another distance on $X$ by setting

$$
d_{b}(x, y)=\inf _{\gamma} l(\gamma)
$$

where $\gamma$ runs over every $b$-chains $x=x_{0} \ldots x_{m}=y$ and where $l(\gamma)=$ $\sum_{i=1}^{m} d\left(x_{i}, x_{i-1}\right)$ is the length of $\gamma$.

Let us introduce various natural notions of geodesicity.

Definition 6.1. We say that a metric space $(X, d)$ is

- $b$-geodesic if $d(x, y)$ equals the minimal length of a $b$-chain between $x$ and $y$, or equivalently if $d=d_{b}$.

- quasi-geodesic if there exists $b>0$ such that the identity map $(X, d) \rightarrow$ $\left(X, d_{b}\right)$ is a quasi-isometry;

- coarsely geodesic if there exists $b>0$ such that $\left(X, d_{b}\right) \rightarrow(X, d)$ is a uniform embedding.

Being coarsely geodesic is actually equivalent to being large-scale uniformly connected (see [30]): a space $X$ is large-scale uniformly connected if there exists $b>0$ such that every $x, y \in X$ can be connected by a $b$-chain whose length only depends on $d(x, y)$.

Clearly, being coarsely geodesic is preserved by large-scale equivalence.

Proposition 6.2. A metric measure space with locally doubling Property admits a discretization if and only if it is coarsely geodesic. Moreover $X$ is quasi-isometric to a graph if and only if it is quasi-geodesic.

Proof. Assume that $X=(X, d, \mu)$ is metrically proper, coarsely geodesic and locally doubling. Consider a minimal covering of $X$ with balls of radius $h$. We construct a weighted graph $G(X)$ as follows; the vertices of $G(X)$ are the centers of the balls; we put an edge between two vertices if the balls intersect. Since $X$ is coarsely geodesic, $G(X)$ is connected as soon as $h$ is large enough. Moreover, coarse geodesicity and locally doubling Property imply that the injection map $G(X) \hookrightarrow X$ is a large-scale equivalence. The converse is obvious.

\subsection{Locally compact groups}

Let $G$ be a group. Recall that a length function on $G$ is function $L: G \rightarrow \mathbb{R}_{+}$ such that $L(1)=0$ and

$$
\forall g, h \in G, \quad L(g h) \leq L(g)+L(h) .
$$


If $L$ is a length function, then $d(g, h)=L\left(g^{-1} h\right)$ defines a left-invariant pseudo-metric on $G$. Conversely, if $d$ is a left-invariant pseudo-metric on $G$, then $L(g)=d(1, g)$ defines a length function on $G$.

Definition 6.3. Let $G$ be a locally compact group. A metric $d$ on $G$ is called uniform if for any of sequence $\left(g_{n}, h_{n}\right) \in(G \times G)^{\mathbb{N}}, d\left(g_{n}, h_{n}\right) \rightarrow \infty$ if and only if $g_{n}^{-1} h_{n}$ leaves every compact eventually.

By the Birkhoff-Kakutani metrization theorem [18, Theorem 7.2], $G$ admits uniform left-invariant metrics if and only if $G$ is $\sigma$-compact. The following proposition is straightforward and left to the reader.

Proposition 6.4. Let $(X, d, \mu)$ and $(Y, d, \mu)$ be a metric measure space and let $G$ be a locally compact group acting properly and co-compactly by isometries that preserve the measure. Then $X$ is locally doubling and $X$ and $Y$ are large-scale equivalent.

Corollary 6.5. Let $d$ and $d^{\prime}$ be two uniform metrics on $G$. The spaces $(G, d)$ and $\left(G, d^{\prime}\right)$ are doubling at any (large enough) scale and the identity map $(G, d) \rightarrow\left(G, d^{\prime}\right)$ is a large-scale equivalence.

Proposition 6.6. Let $G$ be a locally compact group. The following statements are equivalent.

(i) G admits a uniform, coarsely geodesic metric;

(ii) $G$ admits a uniform, quasi-geodesic metric;

(iii) $G$ admits a left-invariant, proper, quasi-geodesic metric;

(iv) $G$ admits a left-invariant proper metric, quasi-isometric to a graph with bounded degree;

(v) $G$ is compactly generated.

Proof. Clearly, $($ iii $) \Rightarrow(i i) \Rightarrow(i)$ are obvious, $(i i i) \Leftrightarrow(i v)$ results from Proposition 6.2. Let us prove that $(v) \Rightarrow(i v)$. Assume that $G$ is compactly generated and let $S$ be a compact symmetric subset $S$. One can equip $G$ with a uniform quasi-geodesic length function setting

$$
\forall g \in G, \quad|g|_{S}=\inf \left\{n \in \mathbb{N}, g \in S^{n}\right\} .
$$

Now, let us prove that $(i) \Rightarrow(v)$. Suppose that $G$ has a uniform, coarsely geodesic metric $d$ with constant $C$. Since $d$ is uniform, there exists $R<\infty$ such that for all $g \in G$, the closed ball $B(g, C)$ is compact and contained in $g \cdot B(1, R)$.

We claim that $G$ is generated by $B(1, R)$. Fix $g \in G$. Indeed, let $g_{1}=$ $1, \ldots, g_{n}=g$ be a chain such that $d\left(g_{i}, g_{i+1}\right) \leq C$ for every $1 \leq i \leq n-1$. We have $g_{i+1} \in B\left(g_{i}, C\right) \subset g_{i} \cdot B(1, R)$. Hence, an immediate induction shows that $g \in B(1, R)^{n}$ and we are done. 


\section{Equivalence of Sobolev inequalities with respect to different gradients}

Here, we show that large-scale Sobolev inequalities do not really depend on the kind of gradient that we use to write them. In spite of its easy and short proof, this result is crucial for our purpose since it shows that our definitions are natural.

The following proposition is an immediate consequence of the definitions.

Proposition 7.1. If $h^{\prime} \geq h>0$, then

$$
\left\||\nabla f|_{h^{\prime}}\right\|_{p} \geq\left\||\nabla f|_{h}\right\|_{p}
$$

Moreover, if $P$ is a viewpoint at scale $h$ with constants $c$ and $A$ (see the definition below) and if $q \leq q^{\prime} \leq \infty$, then

$$
c|\nabla f|_{h, q} \leq|\nabla f|_{P, q} \leq|\nabla f|_{P, q^{\prime}} \leq|\nabla f|_{A h} \quad \forall f \in L^{\infty}(X) .
$$

The non-trivial comparisons between different gradients are summarized in the following proposition.

Proposition 7.2. Let $X$ be some metric measure space satisfying a Sobolev inequality $\left(S_{\varphi}^{p}\right)$ at scale $h$. Then, for any viewpoint $P=\left(P_{x}\right)_{x \in X}$ at scale $2 h, X$ satisfies $\left(S_{\varphi}^{p}\right)$ w.r.t. $|\nabla|_{P, q}$ for any $q \geq 1$.

Proof. By Proposition 7.1, it suffices to prove that $X$ satisfies $\left(S_{\varphi}^{p}\right)$ w.r.t. $|\nabla|_{2 h, 1}$. Write

$$
P_{x}=\frac{1}{V(x, h)} 1_{B(x, h)} \quad \forall x \in X .
$$

For every $f \in L^{\infty}(X)$ we write

$$
P f(x)=\int f d P_{x}, \quad \forall x \in X .
$$

Lemma 7.3. There exists $C<\infty$ such that

$$
|\nabla P f|_{h}(x) \leq C|\nabla f|_{h, 1}(x) \quad \forall f \in L^{\infty}(X), \forall x \in X
$$

Proof. Consider some $y \in B(x, h)$.

$$
|P f(x)-P f(y)| \leq|P f(x)-f(x)|+|P f(y)-f(x)| \leq C|\nabla|_{2 h, 1} f(x) .
$$

with $C<\infty$ depending only on the doubling constant at scale $h$. 
Now apply the Sobolev inequality $\left(S_{\varphi}^{p}\right)$ at scale $h$ to $P f$,

$$
\left\||\nabla P f|_{h}\right\|_{p} \geq \varphi^{-1}(\Omega)\|P f\|_{p} \geq \varphi^{-1}(\Omega)\|f\|_{p}-\varphi^{-1}(\Omega)\left|\|f\|_{p}-\|P f\|_{p}\right| .
$$

Now, if $\left\||\nabla f|_{h, 1}\right\|_{p} \geq\|f\|_{p} / 2$, there is nothing to prove. Hence, assuming the contrary, and since $\left|\|f\|_{p}-\|P f\|_{p}\right| \leq\left\||\nabla f|_{h, 1}\right\|_{p}$, we obtain

$$
\left\||\nabla P f|_{h}\right\|_{p} \geq \varphi^{-1}(\Omega)\|f\|_{p} / 2,
$$

which yields

$$
\left\||\nabla f|_{h, 1}\right\|_{p} \leq C^{-1} \varphi^{-1}(\Omega)\|f\|_{p} / 2
$$

thanks to the lemma.

\section{Invariance of Sobolev inequalities under large-scale equivalence}

The aim of this section is to prove the following theorem.

Theorem 8.1. Let $F: X \rightarrow X^{\prime}$ be a large-scale equivalence between two spaces $X$ and $X^{\prime}$ satisfying the locally doubling property. Assume that for $h>0$ fixed, the space $X$ satisfies a Sobolev inequality $\left(S_{\varphi}^{p}\right)$ at scale $h$, then there exists $h^{\prime}$, only depending on $h$ and on the constants of $F$ such that $X^{\prime}$ satisfies $\left(S_{\varphi}^{p}\right)$ at scale $h^{\prime}$. In particular, large-scale Sobolev inequalities are invariant under large-scale equivalence.

Before proving Theorem 8.1, we will need a few preliminary technical results.

\subsection{Thick subsets}

Definition 8.2. A subset $A$ of a metric space is called $h$-thick if it is a reunion of closed balls of radius $h$.

Roughly speaking, the following proposition says that we can focus on functions with thick support.

Proposition 8.3. Let $X=(X, d, \mu)$ be a metric measure space. Fix some $h>0$ and some $p \in[1, \infty]$. There exists a constant $C>0$ such that for any $f \in L^{\infty}(X)$, there is a function $\tilde{f} \in L^{\infty}(X)$ whose support is included in a $h / 2$-thick subset $\Omega$ such that

$$
\mu(\Omega) \leq \mu(\operatorname{Supp}(f))+C
$$

and for every $p \in[1, \infty]$,

$$
\frac{\left\||\nabla \tilde{f}|_{h / 2}\right\|_{p}}{\|\tilde{f}\|_{p}} \leq C \frac{\left\||\nabla f|_{h}\right\|_{p}}{\|f\|_{p}}
$$


Proof. Let us prove the proposition for $p<\infty$. Let $f \in L^{\infty}(X)$ be such that $\|f\|_{p}=1$. Assume that $f$ satisfies

$$
\left\||\nabla|_{h} f\right\|_{p} \geq \frac{1}{2}
$$

Then, for $\tilde{f}$, consider for instance the indicator function of a ball $B(x, a)$ of volume 1 (so that $\|\tilde{f}\|_{p}=1$ ). We have

$$
\left\||\nabla \tilde{f}|_{h / 2}\right\|_{p}^{p} \leq \mu(B(1+h / 2)) \leq C \mu(B(x, a))=C .
$$

Thus, let us assume that

$$
\left\||\nabla f|_{h}\right\|_{p} \leq \frac{1}{2}
$$

Let $\Omega$ be the subset of $\operatorname{Supp}(f)$ defined by

$$
\Omega=\left\{x \in X, d\left(x, \operatorname{Supp}(f)^{c}\right) \geq h / 2\right\}
$$

and set

$$
\tilde{f}=f \cdot 1_{\Omega} .
$$

Note that for every $x \in \operatorname{Supp}(f) \backslash \Omega$, there exists some $y \in B(x, h)$ such that $f(y)=0$. Therefore, we have $|f(x)| \leq|\nabla f|_{h}(x)$. Hence,

$$
\int_{X}|\tilde{f}|^{p} d \mu \geq \int_{X}|f|^{p} d \mu-\int_{X}\left(|\nabla f|_{h}\right)^{p} d \mu \geq \frac{1}{2} .
$$

On the other hand, let $x \in \Omega$. If $d(x, \operatorname{Supp}(f)) \geq h$, then

$$
|\nabla \tilde{f}|_{h / 2}=|\nabla f|_{h / 2} \leq|\nabla|_{h} f
$$

Otherwise,

$$
|\nabla \tilde{f}|_{h / 2} \leq \max \left\{|f(x)|, \sup _{y \in B(x, h / 2)}|f(x)-f(y)|\right\}
$$

and

$$
|\nabla f|_{h}=\sup _{y \in B(x, h)}|f(x)-f(y)|=\max \left\{|f(x)|, \sup _{y \in B(x, h)}|f(x)-f(y)|\right\} .
$$

Thus

$$
|\nabla \tilde{f}|_{h / 2} \leq|\nabla f|_{h}
$$

so we are done.

On the other hand, the locally doubling property "extends" to thick subsets in the following sense. 
Proposition 8.4. Let $X$ be a metric measure space satisfying the locally doubling property. Fix two positive numbers $u$ and $v$. There exists a constant $C=C(u, v)<\infty$ such that for any u-thick subset $A \subset X$, we have

$$
\mu\left([A]_{v}\right) \leq C \mu(A)
$$

Proof. The proof follows from standard covering arguments.

\subsection{Rough volume-preserving property}

Let us prove a useful volume "roughly preserving" property of large-scale equivalences.

Proposition 8.5. Let $X=(X, d, \mu)$ and $X^{\prime}=\left(X^{\prime}, d^{\prime}, \mu^{\prime}\right)$ be two spaces satisfying the locally doubling property and let $F: X \rightarrow X^{\prime}$ be a large-scale equivalence. Let $u>0$, then there exists a constant $C=C(u, F)$ such that

(1) If $A \subset X$ and $A^{\prime} \subset X^{\prime}$ are such that $\left[F^{-1}\left(A^{\prime}\right)\right]_{u} \subset A$, then $\mu^{\prime}\left(A^{\prime}\right) \leq$ $C \mu(A)$.

(2) If $A \subset X$ and $A^{\prime} \subset X^{\prime}$ are such that $[F(A)]_{u} \subset A^{\prime}$, then $\mu(A) \leq$ $C \mu^{\prime}\left(A^{\prime}\right)$.

Proof. Let us prove (1). Let $Z$ be a maximal set of $2 u$-separated points of $F^{-1}\left(A^{\prime}\right)$. Clearly, the balls $(B(z, u))_{z \in Z}$ are disjoint and included in $A$. On the other hand, maximality of $Z$ implies that the family $(B(z, 2 u))_{z \in Z}$ forms a covering of $A$. So we have

$$
\sum_{z \in Z} \mu(B(z, u)) \leq \mu(A) \leq \sum_{z \in Z} \mu(B(z, 2 u))
$$

By property $(a)$ of a large-scale equivalence, there exists $v$ such that for every $x \in X, F(B(x, 2 u)) \subset B(F(x), v)$. In particular, the family $\left((B(F(z), v))_{z \in Z}\right.$ forms a covering of $F(A)$. Using Property (c) of a large-scale equivalence and Doubling Property at any scale of $X$ together with (8.1), we get

$$
\begin{aligned}
\mu\left(A^{\prime}\right) \leq \mu^{\prime}(F(A)) \leq \sum_{z \in Z} \mu^{\prime}(B(F(z), v)) & \leq C^{\prime} \sum_{z \in Z} \mu(B(z, v)) \\
& \leq C \sum_{z \in Z} \mu(B(z, u)) \leq C \mu(A)
\end{aligned}
$$

which proves the proposition. 


\subsection{Proof of the invariance under large-scale equivalence}

Let $F: X \rightarrow X^{\prime}$ be a large-scale equivalence between two spaces $X$ and $X^{\prime}$ satisfying the locally doubling property. Assume that $f \in L^{\infty}\left(X^{\prime}\right)$. For every $h>0$, define a function on $X$

$$
\forall x \in X, \quad \psi_{h}(x)=\sup _{y \in B(x, h)}|f \circ F(y)|
$$

Lemma 8.6. For $h$ large enough, there exists a constant $c=c(h, f)>0$ such that

$$
\mu\left(\left\{\psi_{h}^{p} \geq t\right\}\right) \geq c \mu^{\prime}\left(\left\{|f|^{p} \geq t\right\}\right) .
$$

In particular, for every $p>0$,

$$
\left\|\psi_{h}\right\|_{p} \geq c\|f\|_{p}
$$

Proof. We can obviously assume that $p=1$ and that $f \geq 0$. Thanks to Proposition 8.5, we only have to check that

$$
\left[F^{-1}(\{f \geq t\})\right]_{h} \subset\left\{\psi_{h} \geq t\right\} .
$$

Indeed, let $x \in F^{-1}(\{f \geq t\})$. Then $f \circ F(x) \geq t$. So for all $y \in B(x, h)$, we have $\psi_{h}(y) \geq t$.

Lemma 8.7. For $h^{\prime}$ large enough, there exists a constant $C<\infty$ such that

$$
\mu\left(\left\{\left(\left|\nabla \psi_{h}\right|_{h}\right)^{q}>t\right\}\right) \leq C \mu^{\prime}\left(\left\{\mid\left(\left.\nabla f\right|_{h^{\prime}}\right)^{q}>t / 2\right\}\right) .
$$

In particular, for every $q>0$,

$$
\left\|\left|\nabla \psi_{h}\right|_{h}\right\|_{q} \leq C\left\||\nabla f|_{h^{\prime}}\right\|_{q}
$$

Proof. We can of course assume that $q=1$. Thanks to Proposition 8.5, it suffices to prove that for $h^{\prime}$ large enough,

$$
\left[F\left(\left\{\left|\nabla \psi_{h}\right|_{h}>t\right\}\right)\right]_{h^{\prime} / 2} \subset\left\{|\nabla f|_{h^{\prime}}>t / 2\right\} .
$$

Indeed, let $x \in X$ be such that $\left|\nabla \psi_{h}\right|_{h}(x)>t$. This means that there exists $y \in B(x, h)$ such that $|f \circ F(x)-f \circ F(y)|>t$. On the other hand, by property (a) of a large-scale equivalence, one can choose $h^{\prime}$ such that $d(F(x), F(y)) \leq h^{\prime} / 2$. Hence,

$$
\forall z \in B\left(F(x), h^{\prime} / 2\right), \quad|\nabla f|_{h^{\prime}}(z) \geq \max \{|f(x)-z|,|f(y)-z|\} \geq t / 2 .
$$

So $z \in\left\{|\nabla f|_{h^{\prime}}>t / 2\right\}$. 
Lemma 8.8. For $u$ large enough, there exists a constant $C<\infty$ such that

$$
\mu^{\prime}\left(\operatorname{Supp}\left(\psi_{h}\right)\right) \leq C \mu\left([\operatorname{Supp}(f)]_{u}\right) .
$$

Proof. This follows trivially from Proposition 8.5.

Proof of Theorem 8.1. Let $\Omega$ be a compact subset of $X^{\prime}$ of measure $m$. We want to prove that every $f \in L^{\infty}(\Omega)$ satisfies

$$
\|f\|_{p} \leq C \varphi(C m)\left\||\nabla f|_{h}\right\|_{p}
$$

with $h^{\prime}$ and $C$ depending only on $F, h$ and $X$. Thanks to Proposition 8.3 and up to choose a larger $h^{\prime}$, we can assume that $\Omega$ is $v$-thick for any $v>0$. Then, thanks to Lemma 8.8 and to Proposition 8.4, we have

$$
\operatorname{Supp}\left(\psi_{h}\right) \leq C^{\prime} m
$$

for some constant $C^{\prime}$. So apply $\left(S_{\varphi}^{p}\right)$ to $\psi_{h}$ and then conclude thanks to Lemmas 8.6 and 8.7.

\section{Sobolev inequality $\left(S_{\varphi}^{2}\right)$ and on-diagonal upper bounds for random walks}

In this section, we revisit the relations (see [6] for a survey) between Sobolev inequalities $\left(S_{\varphi}^{2}\right)$ and on-diagonal upper bounds for random walks in our general context. The main purpose is to prove a version of [6, Theorem 7.2] (see also [7, Theorem 7.2]) to our more general context.

Theorem 9.1. Let $X=(X, d, \mu)$ be a metric measure space and let $P=$ $\left(P_{x}\right)_{x \in X}$ be a symmetric view-point at scale $h$ on $X$. Let $\varphi$ be some increasing positive function. Define $\gamma$ by

$$
t=\int_{0}^{1 / \gamma(t)}(\varphi(v))^{2} \frac{d v}{v}
$$

(i) Assume that $X$ satisfies a Sobolev inequality $\left(S_{\varphi}^{2}\right)$ w.r.t. $|\nabla f|_{P^{2}, 2}$. Then

$$
p_{x}^{2 n}(x) \leq \gamma(c n) \quad \forall n \in \mathbb{N},
$$

for some constant $c>0$.

(ii) If the logarithmic derivative of $\gamma$ has at most polynomial growth and if

$$
p_{x}^{2 n}(x) \leq \gamma(n) \quad \forall n \in \mathbb{N},
$$

then $X$ satisfies $\left(S_{\varphi}^{2}\right)$ w.r.t. $|\nabla|_{P, 2}$. 
Similarly we have the following version ${ }^{10}$ of $[6$, Theorem 7.1]

Theorem 9.2. Let $X=(X, d, \mu)$ be a metric measure space and let $P=$ $\left(P_{x}\right)_{x \in X}$ be a symmetric view-point at scale $h$ on $X$. Define $\gamma$ by

$$
t=\int_{0}^{1 / \gamma(t)}\left(j_{X, 2}(v)\right)^{2} \frac{d v}{v}
$$

where $j_{X, 2}$ is the isoperimetric profile of $X$ defined with the gradient $|\nabla f|_{P^{2}, 2}$. If the logarithmic derivative of $\gamma$ has at most polynomial growth, then there exists a constant $C>0$ such that

$$
\gamma(C n) \leq \sup _{x \in X} p_{x}^{2 n}(x) \leq \gamma(n) \quad \forall n \in \mathbb{N} .
$$

Proof of Theorem 9.1. In [6, Theorem 7.2], the same result is proved for a weighted graph $(X, \mu)$ using the usual notion of gradient on graphs (see Example 2.4) and where $P$ is the standard random walk on $(X, \mu)$. Their proof only relies on the following formal link between $P$ and the gradient.

$$
c\left(\|f\|_{2}^{2}-\|P f\|_{2}^{2}\right) \leq\|\nabla f\|_{2}^{2} \leq C\left(\|f\|_{2}^{2}-\|P f\|_{2}^{2}\right) .
$$

Here, this relation is satisfied when considering the gradient $|\nabla|_{P^{2}, 2}$ and we even have the equality

Lemma 9.3. For every $f \in L^{2}(X)$, we have

$$
\left\||\nabla f|_{P^{2}, 2}\right\|_{2}^{2}=\|f\|_{2}^{2}-\|P f\|_{2}^{2} .
$$

Proof. We have (see section 2.2)

$$
\begin{aligned}
\left\||\nabla f|_{P^{2}, 2}\right\|_{2}^{2} & =\left\langle\Delta_{P^{2}} f, f\right\rangle \\
& =\left\langle\left(i d-P^{2}\right) f, f\right\rangle \\
& =\|f\|_{2}^{2}-\left\langle P^{2} f, f\right\rangle \\
& =\|f\|_{2}^{2}-\langle P f, P f\rangle
\end{aligned}
$$

So the proof of [6, Theorem 7.2] can be used formally in our context. However, for the sake of completeness, we give a sketch of this proof. First, using that $P^{n}$ is symmetric, one checks easily that

$$
\sup _{x \in X} p_{x}^{2 n}(x)=\left\|P^{2 n}\right\|_{1 \rightarrow \infty}
$$

where $\|\cdot\|_{p \rightarrow q}$ denotes the operator norm form $L^{p}(X, \mu)$ to $L^{q}(X, \mu)$.

\footnotetext{
${ }^{10}$ The proofs are straightforward adaptations of their versions for graphs in [6] so we will only prove Theorem 9.1 .
} 
Proof of (i). Assume that $\left(S_{\varphi}^{2}\right)$ holds. Let us start with an important lemma.

Lemma 9.4. The Sobolev inequality $\left(S_{\varphi}^{2}\right)$ for the $L^{2}$-gradient w.r.t. the viewpoint $P$ is equivalent to the so-called Nash inequality

$$
\|f\|_{2}^{2} \leq C \varphi^{2}\left(C \frac{\|f\|_{1}^{2}}{\|f\|_{2}^{2}}\right)\left\||\nabla|_{P, 2}\right\|_{2}^{2}
$$

Proof. Assume that a function $f$ satisfies Nash inequality. Using Schwarz inequality and the fact that $\varphi$ is nondecreasing, we obtain

$$
\|f\|_{2}^{2} \leq \varphi^{2}\left(\frac{\|f\|_{2}^{2}}{\|f\|_{1}^{2}}\right)\left\||\nabla f|_{P^{2}, 2}\right\|_{2}^{2} \leq \varphi^{2}(|\Omega|)\left\||\nabla f|_{P^{2}, 2}\right\|_{2}^{2}
$$

The proof of the other implication relies on an argument of Grigor'yan in [12]. Assume that $\left(S_{\varphi}^{2}\right)$ holds. Let $f \in L^{\infty}(X)$. For every $\lambda>0$, since $f<2(f-\lambda)$ on $\{f>2 \lambda\}$, we may write

$$
\begin{aligned}
\int f^{2} & =\int_{f>2 \lambda} f^{2}+\int_{f \leq 2 \lambda} f^{2} \\
& \leq 4 \int_{f>2 \lambda}(f-\lambda)^{2}+2 \lambda \int_{f \leq 2 \lambda} f \\
& \leq 4 \int_{f>2 \lambda}(f-\lambda)^{2}+2 \lambda\|f\|_{1}
\end{aligned}
$$

Now applying $\left(S_{\varphi}^{2}\right)$ to $(f-\lambda)_{+}$gives

$$
\int(f-\lambda)_{+}^{2} \leq \varphi^{2}(\mu(\{f>2 \lambda\}))\left\||\nabla f|_{P^{2}, 2}\right\|_{2}^{2}
$$

that is, since

$$
\mu(\{f>\lambda\}) \leq \frac{\|f\|_{1}}{\lambda}
$$

and $\varphi$ is non-decreasing,

$$
\int(f-\lambda)_{+}^{2} \leq \varphi^{2}\left(\frac{\|f\|_{1}}{\lambda}\right)\left\||\nabla f|_{P^{2}, 2}\right\|_{2}^{2} .
$$

Therefore

$$
\int f^{2} \leq 4 \varphi^{2}\left(\frac{\|f\|_{1}}{\lambda}\right)\left\||\nabla f|_{P^{2}, 2}\right\|_{2}^{2}+2 \lambda\|f\|_{1} .
$$

Letting $\varepsilon>0$ and taking $\lambda=\varepsilon\|f\|_{2}^{2} /\|f\|_{1}$ in this equation yields

$$
\|f\|_{2}^{2} \leq 4 \varphi^{2}\left(\frac{\|f\|_{2}^{2}}{\varepsilon\|f\|_{1}^{2}}\right)\left\||\nabla f|_{P^{2}, 2}\right\|_{2}^{2}+2 \varepsilon\|f\|_{2}^{2}
$$


or equivalently,

$$
\|f\|_{2}^{2} \leq \frac{4}{1-2 \varepsilon} \varphi^{2}\left(\frac{\|f\|_{2}^{2}}{\varepsilon\|f\|_{1}^{2}}\right)\left\||\nabla f|_{P^{2}, 2}\right\|_{2}^{2}
$$

Taking $\varepsilon=1 / 4$, for example yields

$$
\|f\|_{2}^{2} \leq 8 \varphi^{2}\left(4 \frac{\|f\|_{2}^{2}}{\|f\|_{1}^{2}}\right)\left\||\nabla f|_{P^{2}, 2}\right\|_{2}^{2}
$$

which is the expected Nash inequality.

Now, consider $f \in L^{1}(X, \mu)$, non-negative, with $\|f\|=1$ and define a sequence $u_{n}=\left\|P^{n} f\right\|_{2}^{2}$. The above inequality applied to the function $P^{n} f$ thus reads as

$$
u_{n} \leq \varphi^{2}\left(1 / u_{n}\right)\left(u_{n}-u_{n+1}\right)
$$

since $\left\|P^{n} f\right\|_{1}=\|f\|_{1}=1$ by Markov property of $P$. Let $t \rightarrow u_{t}$ be the increasing, piecewise linear function extending $u_{n}$ on $\mathbb{R}_{+}$. If we put $v_{t}=1 / u_{t}$, then the above inequality becomes

$$
d t \leq \varphi^{2}\left(v_{t}\right) \frac{d v_{t}}{v_{t}}
$$

hence, by integrating between 0 and $t$, we obtain

$$
t \leq \int_{v_{0}}^{1 / v_{t}} \varphi^{2}(s) \frac{d s}{s}
$$

and since by definition

$$
t=\int_{0}^{1 / \gamma(t)}(\varphi(v))^{2} \frac{d v}{v}
$$

this means that $\gamma(t) \leq v_{t}$, i.e.

$$
\left\|P^{n} f\right\|_{2}^{2} \leq \gamma(n)
$$

from which we deduce

$$
\left\|P^{n}\right\|_{1 \rightarrow 2} \leq \sqrt{\gamma(n)}
$$

Now, using the fact that $P^{n}$ is symmetric,

$$
\left\|P^{n}\right\|_{2 \rightarrow \infty}=\left\|P^{n}\right\|_{1 \rightarrow 2} \leq \sqrt{\gamma(n)} .
$$

Hence

$$
\left\|P^{2 n}\right\|_{1 \rightarrow \infty} \leq\left\|P^{n}\right\|_{2 \rightarrow \infty}\left\|P^{n}\right\|_{1 \rightarrow 2} \leq \gamma(n) .
$$

So (i) follows. 
Proof of (ii). Assume that the decay $\left\|P^{2 n}\right\|_{1 \rightarrow \infty} \leq \gamma(n)$ holds. Observe that $\left\|P^{2 n}\right\|_{1 \rightarrow \infty}=\left\|P^{n}\right\|_{1 \rightarrow 2}$, then take $f$ with $\|f\|_{1}=1$ and define as above $u_{n}=\left\|P^{n} f\right\|_{2}^{2}$. Since $P$ is self-adjoint,

$$
\left\|P^{n} f\right\|_{2}^{2}=\left\langle P^{n} f, P^{n} f\right\rangle=\left\langle P^{n-1} f, P^{n+1} f\right\rangle \leq\left\|P^{n-1} f\right\|_{2}\left\|P^{n+1} f\right\|_{2} .
$$

In other words, $u_{n}^{2} \leq u_{n-1} u_{n+1}$ and $u_{n+1} / u_{n}$ is nondecreasing in $n$. It follows that

$$
\left(\frac{u_{1}}{u_{0}}\right)^{n} \leq \frac{u_{1}}{u_{0}} \frac{u_{2}}{u_{1}} \ldots \frac{u_{n}}{u_{n-1}}=\frac{u_{n}}{u_{0}}
$$

Now, since by assumption $u_{n} \leq \gamma(n)$,

$$
\log \frac{\|f\|_{2}^{2}}{\gamma(n)} \leq \log \frac{u_{0}}{u_{n}} \leq n \log \frac{u_{0}}{u_{n}} \leq n\left(\frac{u_{0}}{u_{1}}-1\right),
$$

hence

$$
\|P f\|_{2}^{2} \leq\left(\frac{n}{\log \frac{\|f\|_{2}^{2}}{\gamma(n)}}\right)\left(\|f\|_{2}^{2}-\|P f\|_{2}^{2}\right), \forall n \in \mathbb{N} .
$$

Finally, for all $f$ such that $\|f\|_{1}=1$,

$$
\|f\|_{2}^{2} \leq\left(\frac{n}{\log \frac{\|f\|_{2}^{2}}{\gamma(n)}}+1\right)\left(\|f\|_{2}^{2}-\|P f\|_{2}^{2}\right), \forall n \in \mathbb{N} .
$$

An optimization ${ }^{11}$ in $n$ yields the Nash inequality that is equivalent to $\left(S_{\varphi}^{2}\right)$ by Lemma 9.4.

\section{Controlling the scale of Sobolev Inequalities}

\subsection{Going down the scale}

In this section, we address the following question. Let $X=(X, d, \mu)$ be a metric measure space $X$ satisfying a Sobolev inequality at scale $h$; we know that it automatically satisfies the same Sobolev inequalities at any larger scale; but under what assumptions does it satisfy this inequality at some smaller scale $h^{\prime}$ ? This can be compared to a similar discussion in [30] where we considered the isoperimetric properties of a metric measure space ${ }^{12}$.

For example, consider $X=\mathbb{Z}^{d}(d \geq 2)$ equipped with the distance $d(x, y)=\sum_{i=1}^{d}\left|y_{i}-x_{i}\right|$ and with the countable measure. It is well known that $X$ satisfies a Sobolev inequality $S(d /(d-1), 1)$ at any scale $\geq 1$. But no

\footnotetext{
${ }^{11}$ This is where condition $(\delta)$ is needed.

${ }^{12}$ This is a particular case of the present discussion corresponding to $p=1$.
} 
Sobolev inequality is available at a scale $s<1$ since for every $f \in L^{\infty}(X)$, $|\nabla f|_{s}=0$. Clearly, the problem comes from the lack of connectivity at scale $<1$.

The following proposition shows that Property ${ }^{13}$ of coarse $b$-geodesicity (also called uniform $b$-connectedness) together with Property of locally doubling are sufficient to control the minimal scale at which Sobolev inequalities may be valid.

Proposition 10.1. Assume that $X$ is a coarse b-geodesic space satisfying the locally doubling property $r \geq b$. Then $X$ satisfies a large-scale Sobolev inequality if and only if it satisfies the same Sobolev inequality at scale $2 b$ (but with different constants). In other words, the asymptotic behavior of the isoperimetric profile $j_{X, p}$ does not depend on the scale, provided it is larger than $2 b$.

Proof. Let $f \in L^{\infty}(X)$. Let us prove that for all $h \geq 2 b$, there is a constant $C=C(h)<\infty$ such that for every $t>0$

$$
\mu\left(\left\{|\nabla f|_{h}>t\right\}\right) \leq C \mu\left(\left\{|\nabla f|_{2 b}>t / C\right\}\right) .
$$

Consider a point $x \in\left\{|\nabla f|_{h}>t\right\}$ : there is $y \in B(x, h)$ such that $\mid f(x)-$ $\varphi(y) \mid>t$. Now, let $x=x_{1} \ldots x_{m}=y$ be a $b$-connecting chain between $x$ and $y$ (with $m$ only depending on $h$ ). Clearly, there exists $1 \leq i<m$ such that $\left|\varphi\left(x_{i}\right)-\varphi\left(x_{i+1}\right)\right|>t / m$. So in particular, for all $z \in B\left(x_{i}, b\right)$, $|\nabla f|_{2 b}(z)>t /(2 m)$. Let $Z$ be a maximal $2 E$-separated subset of $\left\{|\nabla f|_{h}>\right.$ $t\}$. The balls $(B(z, 2 E))_{z \in Z}$ form a covering of $\left\{|\nabla f|_{h}>t\right\}$. On the other hand, by the previous discussion, in each ball $B(z, E)$, one can find a ball $B\left(x_{z}, b\right)$ included in $\left\{|\nabla f|_{2 b}>t /(2 m)\right\}$. Since the balls $\left(B\left(x_{z}, b\right)\right)_{z \in Z}$ are disjoint, (10.1) follows from locally doubling property $r \geq b$.

As an interesting corollary of Proposition 10.1 and Proposition 6.6, we obtain that if $h$ is large enough, a Sobolev inequality is satisfied at scale $h$ on a locally compact compactly generated group if and only if it is satisfied at large scale. It also allows to define an $L^{p}$-isoperimetric profile on locally compact compactly generated groups, whose asymptotic behavior does not depend on the scale, provided it is large enough. As a corollary of Theorem 8.1, we therefore have

Corollary 10.2. Let $H$ and $G$ be quasi-isometric amenable unimodular locally compact compactly generated group. Then,

(1) $j_{H, p} \approx j_{G, p}$;

(2) $J_{H, p}^{b} \approx J_{G, p}^{b}$.

\footnotetext{
${ }^{13}$ see definition 6.1 .
} 
Remark 10.3. In particular, for $p=2$, the asymptotic behavior when $r \rightarrow \infty$ of first eigenvalue $\delta_{P}(r)$ of the Laplacian associated to any viewpoint $P$ whose support generates the group, acting on square-integrable functions supported in balls of radius $r$, does not depend on $P$. We therefore denote it by $\delta_{G}$. Part (2) of Corollary 10.2 for $p=2$ says that the asymptotic behavior of $\delta_{G}$ is invariant under quasi-isometry (see Section 2.2).

\subsection{From finite scale to infinitesimal scale}

Definition 10.4. (see for instance [28, Definition 1.18]) Let $(X, d)$ be a metric space, and let $u$ and $g$ be two Borel measurable functions defined on $X$, with $u$ real-valued and $g$ taking values in $[0, \infty]$. We say that $g$ is an generalized gradient of $u$ if

$$
|u(\gamma(a))-u(\gamma(b))| \leq \int_{a}^{b} g(\gamma(t)) d t
$$

whenever $a, b \in \mathbb{R}$ and $\gamma:[a, b] \rightarrow X$ is 1 -Lipschitz (so that $d(\gamma(s), \gamma(t)) \leq$ $|s-t|$ for all $s, t \in[a, b])$.

Example 10.5. [28, Lemma 1.20] The function $g$ defined by

$$
g(x)=\liminf _{r \rightarrow 0} r^{-1} \sup _{y \in B(x, r)}|u(y)-u(x)|
$$

is a generalized gradient of $u$. Let us call $g$ the standard upper gradient of $u$ and we denote it by $|\bar{\nabla} u|$.

The following proposition is obvious by passing to the limit.

Proposition 10.6. Fix $p \in[1, \infty]$. Assume that for every $h>0,(X, d, \mu)$ satisfies a Sobolev inequality $\left(S_{\varphi}^{p}\right)$ w.r.t. the gradient $|\nabla|_{h}$. Suppose that the constants appearing in these inequalities are uniform with respect to $h$, then $X$ satisfies $\left(S_{\varphi}^{p}\right)$ w.r.t. the standard upper gradient.

The following fact had already been noticed in the case of a discretization of a manifold [10]. Its proof, here, is straightforward from the definition of $|\nabla|_{P, p}$.

Proposition 10.7. Fix some $h>0$ and $p \in[1, \infty]$. Let $(X, d, \mu)$ be a metric measure space with doubling property at radius $\geq h$, and let $P$ be a viewpoint at scale $h$ on $X$. Suppose that a function $u \in L^{\infty}(X)$ satisfies $\left(S_{\varphi}^{p}\right)$ w.r.t. $|\nabla|_{P, p}$. Let $g$ be an generalized gradient of $u$. We assume that $u$ satisfies the following local Poincaré inequality $(P(1, p))_{l o c}$

$$
\int_{B(x, h)}|h(y)-h(x)|^{p} d P_{x}(y) \leq C \int_{B\left(x, h^{\prime}\right)} g^{p}(y) d \mu(y)
$$

for some constants $C, h<\infty$. Then u satisfies $\left(S_{\varphi}^{p}\right)$ w.r.t. $g$. 
Example 10.8. Let $M$ be a Riemannian manifold. Then the local norm of its usual gradient trivially coincides with the standard upper gradient on $M$. Now, assume that $M$ satisfying a local Poincaré inequality (as in the Proposition) and let $X$ be a discretization of $M$. According to Theorem 8.1, if $X$ satisfies $\left(S_{\varphi}^{p}\right)$, then $M$ also satisfies $\left(S_{\varphi}^{p}\right)$ w.r.t. its usual gradient.

\subsection{From infinitesimal scale to finite scale}

In this last section, we will prove that if a metric measure space satisfies a Sobolev inequality w.r.t. the standard upper gradient (see Example 10.5), then it satisfies this Sobolev inequality at any scale.

Theorem 10.9. Fix $p \in[1, \infty]$. Let $(X, d, \mu)$ be a metric measure space satisfying the locally doubling property. Assume that $(X, d, \mu)$ satisfies a Sobolev inequality $\left(S_{\varphi}^{p}\right)$ w.r.t. the standard upper gradient $|\bar{\nabla}|$. Then $X$ satisfies $\left(S_{\varphi}^{p}\right)$ w.r.t. $|\nabla|_{h}$ for every $h>0$.

Proof. Assume that $X$ satisfies $\left(S_{\varphi}^{p}\right)$ w.r.t. the standard upper gradient. Using the same tools as in the proof of Proposition 7.2, one can see that it suffices to show that for every $h>0$ and every function $f$, there exists a viewpoint $P$ at scale $h / 2$ such that

$$
\|P f\|_{p} \leq C \varphi(\mu(\Omega))\left\||\nabla P f|_{h}\right\|_{p}
$$

where $\Omega$ is a measurable subset containing the support of $f$. According to Proposition 8.3, we can assume that $\operatorname{Supp}(f)$ is thick. Thus, thanks to Proposition 8.4 , we can replace $\Omega$ by $[\Omega]_{A h}$ that ${ }^{14}$ contains $\operatorname{Supp}(P f)$. Finally, it suffices to prove that $\left(S_{\varphi}^{p}\right)$ w.r.t. $|\nabla|_{h}$ is satisfied for functions of the form $P f$, with $f \in L^{\infty}(X)$.

Define a 1-Lipschitz map $\theta: X \times X \rightarrow \mathbb{R}_{+}$by $\theta(x, y)=d\left(y, B(x, h)^{c}\right)$. Write

$$
p_{x}(y)=\frac{\theta(x, y)}{K(x)}
$$

where

$$
K(x)=\int_{B(x, h)} \theta(x, z) d \mu(z) .
$$

Since $X$ is locally doubling, one can easily check that $p_{x}(y)$ is the density of a viewpoint $P$ at scale $h$. Moreover, $D^{-1} V(x, h) \leq K\left(x^{\prime}\right) \leq D V(x, h)$ where $D \geq 1$ only depends on the doubling constant at scale $h$.

\footnotetext{
${ }^{14} \mathrm{~A}$ is the large constant appearing in the definition of a viewpoint at scale $h$.
} 
Let $x^{\prime}$ be a point distinct from $x$. We have

$$
\begin{array}{rl}
P & f\left(x^{\prime}\right)-P f(x)=\int_{X}\left(p_{x^{\prime}}(y)-p_{x}(y)\right) f(y) d \mu(y) \\
& =\int_{X}\left(p_{x^{\prime}}(y)-p_{x}(y)\right)(f(y)-f(x)) d \mu(y) \\
& =\int_{X} \frac{\theta\left(x^{\prime}, y\right) K(x)-\theta(x, y) K\left(x^{\prime}\right)}{K(x) K\left(x^{\prime}\right)}(f(y)-f(x)) d \mu(y) \\
& =\int_{X} \frac{\left(\theta\left(x^{\prime}, y\right)-\theta(x, y)\right) K(x)-\theta(x, y)\left(K\left(x^{\prime}\right)-K(x)\right)}{K(x) K\left(x^{\prime}\right)}(f(y)-f(x)) d \mu(y)
\end{array}
$$

Since $X$ is locally doubling, it is not difficult to see that for $x^{\prime}$ closed to $x$, $C^{-1} K(x) \leq K\left(x^{\prime}\right) \leq C K(x)$ where $C \geq 1$ only depends on the doubling constant at scale $h$. Hence,

$$
|\bar{\nabla} P f|(x) \leq C \int_{X} \frac{\left|\bar{\nabla}_{x} \theta\right|(x, y) K(x)+\theta(x, y)|\bar{\nabla} K|(x)}{K(x)^{2}}|f(y)-f(x)| d \mu(y)
$$

On the other hand, note that

$$
|\bar{\nabla} K|(x) \leq \int_{X}\left|\bar{\nabla}_{x} \theta\right|(x, z) d \mu(z) \leq V(x, h) .
$$

Up to change the constant $C$, we conclude that

$$
\begin{aligned}
|\bar{\nabla} P f|(x) & \leq C \frac{1}{V(x, h)} \int_{B(x, h)}|f(y)-f(x)| d \mu(y) \\
& \leq C|\nabla f|_{h}(x) .
\end{aligned}
$$

Now, to conclude, it remains to apply $\left(S_{\varphi}^{p}\right)$ w.r.t. the standard upper gradient to $P f$. Together with the above inequality, we obtain (10.2).

Corollary 10.10. If a Riemannian manifold $M$ with locally doubling property satisfies $\left(S_{\varphi}^{p}\right)$ for the usual gradient, then it satisfies it at any scale. If $X$ is a discretization of $M$, then it also satisfies $\left(S_{\varphi}^{p}\right)$.

Remark 10.11. Assume that $X$ is coarsely $b$-geodesic for every $b>0$ (e.g. $X$ is a Riemannian manifold), so that Proposition 10.1 applies. Note that in the proof of Theorem 10.9, we actually show that a Sobolev inequality at large scale is equivalent to the Sobolev inequality for the standard upper gradient restricted to functions of the form $g=P f$, where $P$ is a viewpoint at some positive scale. 


\section{Applications to quasi-transitive spaces}

\subsection{Existence of a spectral gap on a quasi-transitive metric mea- sure space}

\section{The main result}

Definition 11.1. A quasi-transitive measure space $(X, \mu)$ is a locally compact Borel measure space on which a locally compact group $G$ acts measurably, co-compactly, properly, and almost preserving the measure $\mu$, i.e.

$$
\sup _{g \in G} \sup _{x \in X} \frac{d(g \cdot \mu)}{d \mu}(x)<\infty .
$$

Definition 11.2. We call a metric measure space $(X, d, \mu)$ a quasi-transitive metric measure space if $(X, \mu)$ is a quasi-transitive measure space and if $d$ is a $G$-invariant metric on $X$ which is proper and finite on compact sets (note that $d$ is not supposed to be continuous on $X$ ).

Proposition 11.3. If $G$ is $\sigma$-compact, then every $G$-quasi-transitive measure space $(X, \mu)$ can be equipped with a metric d such that $(X, d, \mu)$ is a quasi-transitive metric measure space.

Proof. We start with a proper $G$-invariant metric on $G$ [18, Theorem 7.2]. Take a fundamental domain $D$ in $X$ relative to the $G$-action. As the action is co-compact, we can assume that $D$ is relatively compact. Let $K$ be the intersection of all stabilizers of elements of $D$. As the action is proper, $K$ is a compact subgroup of $G$. Consider the $G$-invariant metric on $G / K$ obtained, first by averaging our metric on $G$ over $K$ (i.e. replacing it by $\left.\int_{K} d(g k, h k) d k\right)$, and then by pushing the corresponding bi- $K$-invariant metric to $G / K$. We have a natural map $\alpha: X \rightarrow G / K$, where $\alpha(x)$ is the unique $g K$ such that $x \in g D$. Pulling the metric of $G / K$ to $X$ yields a $G$-invariant pseudo-metric on $X$ which is proper and finite on compact sets. To obtain a true metric, one can for instance add the discrete metric on $X$ (i.e. such that two distinct points are at distance 1 ).

Theorem 2 now appears as a corollary of

Theorem 11.4. Let $G$ be a locally compact group and let $(X, d, \mu)$ be a quasi$G$-transitive metric measure space. Then $G$ is unimodular and amenable if and only if for $h$ large enough (resp. for any $h$ ) and every reversible viewpoint $P$ at scale $h$ on $(X, d, \mu)$, the spectral radius $\rho(P)=1$, or in other words, if the discrete Laplacian $\Delta=I-P$ has no spectral gap around zero.

Proof. The proof splits into three parts. First, by Theorem 3.5, one checks easily that $\rho(P)=1$ if and only if the large-scale profile $j_{X, 2}(t) \rightarrow \infty$ when $t \rightarrow \infty$. Indeed, $j_{X, 2}(t) \leq C$ means that $X$ satisfies a large-scale Sobolev 
inequality $\left(S_{\varphi}^{2}\right)$ with $\varphi(t)=C$. Thus by Theorem 3.5, this happens if and only if $p_{x}^{2 n}(x)$ has exponential decay, i.e. if and only if $\rho(P)<1$.

Second, take a uniform left-invariant metric on $G$. The co-compactness, properness of the $G$-action on $X$, plus the fact that $\mu$ is almost-preserved by $G$ imply that $G$ and $X$ are large-scale equivalent (this is straightforward). Hence, by Theorem 8.1, it is enough to prove Theorem 11.4 for $X=G$. This third step will be achieved by Corollary 11.13.

Remark 11.5. Note that if we assume $G$ compactly generated, then it is classical and not difficult to see that a quasi- $G$-transitive metric measure space is quasi-isometric to $G$, equipped with the word metric $d_{S}$ corresponding to a compact generating subset $S$ of $G$.

Corollary 11.6. Let $M$ be a Riemannian manifold and let $G$ be a closed subgroup of Isom $(M)$ acting co-compactly on $M$. Then $G$ is unimodular and amenable if and only if the spectral radius of the heat kernel on $M$ equals 1 , or in other words, if the (Riemannian) Laplacian on $M$ has no spectral gap around zero.

Proof. The Laplacian has a spectral gap if and only if $M$ satisfies a Sobolev inequality $\|\nabla f\|_{2} \geq c\|f\|_{2}$ for the usual gradient. As $M$ is quasi-transitive, it is easy to check that it satisfies a local Poincaré inequality as in Proposition 10.7. Indeed, one has to prove that such a local Poincaré inequality $(P(1, q))_{\text {loc }}$ holds, for any $q \geq 1$ on a compact subset $K$ such that $X=\cup_{g \in G} g K$. But this results from the fact that such inequality holds in $\mathbb{R}^{d}$. Now, applying Proposition 10.7 and Theorem 10.9, we see that the spectral gap is equivalent to a large-scale Sobolev inequality. We conclude thanks to Theorem 11.4.

\section{Locally compact groups}

All the locally compact groups considered here are $\sigma$-compact. Recall (see Section 6.2 ) that a $\sigma$-compact locally compact group can be endowed with a "large-scale" structure of metric measure space. Let us consider the following natural question: is amenability a geometric property among compactly generated locally compact groups? Recall that a locally compact group is called amenable if it admits a left invariant mean [21]. By geometric property, we mean a property characterized in terms of metric measure space. Moreover, we expect such a property to be invariant under large-scale equivalence. Følner's characterization of amenability implies that the answer is positive when the group is finitely generated. On the opposite, note that any connected Lie group admits a co-compact amenable subgroup (take for instance a maximal solvable subgroup) and therefore is always quasi-isometric 
to a compactly generated locally compact amenable group. So the answer is negative in general. Actually, we will see that the answer is yes if and only if the group is unimodular.

Let $G$ be a $\sigma$-compact locally compact group equipped with some proper left-invariant metric $d$ and with its Haar measure $\mu$. Fix some $h>0$. We define the boundary of a subset $A$ of $G$ by

$$
\partial_{h} A=A B(e, h) \cap A^{c} B(e, h) .
$$

It is important to note that the multiplication by elements of $B(e, h)$ is on the right, so that $A B(e, h)$ has the following metric interpretation:

$$
A B(e, h)=\cup_{x \in A} B(x, h)=[A]_{h}
$$

where $[A]_{h}=\{x \in G, d(x, A) \leq h\}$. In particular, this definition of boundary coincides with the one we gave in introduction for a general metric space.

For any sequence of compact subsets with positive measure $\left(F_{n}\right)$ of $G$ and for every $g \in G$, we define $\phi_{n}(g)=\mu\left(g F_{n} \Delta F_{n}\right) / \mu\left(F_{n}\right)$. Note that here, the multiplication by $g$ is on the left.

Recall [21] that the group $G$ is amenable if and only one of the following equivalent statements holds:

(1) There exists a sequence $\left(F_{n}\right)$ such that $\phi_{n}(g)$ is pointwise converging to zero.

(2) There exists a sequence $\left(F_{n}\right)$ such that $\phi_{n}(g)$ converges to zero uniformly on compact sets.

(3) There exists a sequence $\left(F_{n}\right)$ such that $\mu\left(Q F_{n} \cap Q F_{n}^{c}\right) / \mu\left(F_{n}\right) \rightarrow 0$ for every compact subset $Q$.

If a sequence $\left(F_{n}\right)$ satisfies $(1)$, or equivalently, $(2)$, then it is called a Følner sequence.

Remark 11.7. Generally, in the definition of Følner sequence, $\left(F_{n}\right)$ is also asked to be an increasing exhaustion of $G$ (this also characterizes amenability).

Here, the multiplication by $Q$ is on the left, so that amenability is not a priori characterized in terms of isoperimetry, or in other words, in terms of metric measured space properties. Let us define a geometric version of amenability.

Definition 11.8. The group $G$ is called geometrically amenable if it admits a sequence of compact subsets $\left(F_{n}\right)$ such that one of the following equivalent statements holds:

(1) $\mu\left(F_{n} \triangle F_{n} g\right) / \mu\left(F_{n}\right) \rightarrow 0$ for every $g \in G$.

(2) For every compact subset $Q$ of $G$,

$$
\mu\left(F_{n} Q \cap F_{n}^{c} Q\right) / \mu\left(F_{n}\right) \rightarrow 0 .
$$


Our terminology is justified by the following proposition.

Proposition 11.9. A $\sigma$-compact locally compact group $G$ is geometrically amenable if and only if for $h$ large enough, the isoperimetric profile $j_{G, 1}$ (resp. $j_{G, p}$ for any $p \geq 1$ ) at scale $h$ is unbounded.

Proof. Clearly, (11.1) implies that the large-scale profile $j_{G, 1}$ is unbounded. Conversely, the negation of (11.1) together with the $\sigma$-compacity of $G$ yields the existence of a compact subset $K$ of $G$ such that for every measurable subset $A$ with finite measure,

$$
\mu(A) \leq C \mu(A K \triangle A)
$$

for some constant $C<\infty$. Let $h$ be such that $K \subset B(e, h)$. It follows that

$$
\mu(A) \leq C \mu\left(\partial_{h} A\right),
$$

which means that the profile $j_{X, 1}$ at scale $h$ is bounded.

If $G$ is unimodular, up to replacing $F_{n}$ with $F_{n}^{-1}$, it is equivalent for $G$ to have left or right Følner sequences. In particular, if a group is unimodular, then it is geometrically amenable if and only if it is amenable.

Lemma 11.10. If the group $G$ is non-unimodular, then it satisfies the following isoperimetric inequality for h large enough

$$
\mu\left(\partial_{h} A\right) \geq c \mu(A) \quad \forall A \subset G
$$

where $c$ is some positive constant.

Proof. Let $\delta$ be the modular function of $G$. Since $G$ is non-unimodular, there exists $g \in G$ such that $\delta(g)>1$. So, choosing $h$ large enough, we can assume that $g \in B(e, h)$. Then for any compact subset $A \subset G$, we have

$$
\mu\left(\partial_{h} A\right) \geq \mu(A g \triangle A) \geq \mu(A g)-\mu(A)=(\delta(g)-1) \mu(A) .
$$

Proposition 11.11. Let $G$ be a $\sigma$-compact locally compact group equipped with a left Haar measure. Then $G$ is amenable and unimodular if and only if it admits a geometric Følner sequence. In particular if $G$ is compactly generated, then $G$ is amenable and unimodular if and only if it is geometrically amenable.

Proof. This is a direct consequence of Lemma 11.10 and of the above discussion.

Recall that quasi-isometries between homogeneous metric measure spaces are large-scale equivalences. We have the following corollaries to Theorem 8.1. 
Corollary 11.12. Geometric amenability is invariant under large-scale equivalence between $\sigma$-compact locally compact groups.

Corollary 11.13. Geometric amenability is invariant under quasi-isometry between compactly generated locally compact groups.

The following corollary follows from Propositions 11.11, 6.6 and Theorem 8.1.

Corollary 11.14. A compactly generated locally compact group is not geometrically amenable if and only if it is quasi-isometric to a graph with positive Cheeger constant.

Corollary 11.15. Being amenable and unimodular is invariant under largescale equivalence between $\sigma$-compact locally compact groups.

\section{References}

[1] Brooks, R.: The fundamental group and the spectrum of the Laplacian. Comm. Math. Helv. 56 (1981), 581-598.

[2] Chavel, I.: Riemannian geometry: a modern introduction. Cambridge Tracts in Mathematics 108. Cambridge University Press, Cambridge, 1993.

[3] Chavel, I. and Feldman, E.: Modified isoperimetric constants, and large time heat diffusion in Riemannian manifolds. Duke Math. J. 64 (1991), no. 3, 473-499.

[4] Coulhon, T.: Dimension à l'infini d'un semi-groupe analytique. Bull. Sci. Math. 114 (1990), 485-500.

[5] Coulhon, T.: Espaces de Lipschitz et inégalités de Poincaré. J. Funct. Anal. 136 (1996), 81-113.

[6] Coulhon, T.: Random walks and geometry on infinite graphs. In Lecture notes on analysis on metric spaces (Trento, 1999), 5-36. Appunti Corsi Tenuti Docenti Sc., Scuola Norm. Sup., Pisa, 2000.

[7] Coulhon, T.: Heat kernel and isoperimetry on non-compact Riemannian manifolds. In Heat kernels and analysis on manifolds, graphs and metric spaces (Paris, 2002), 65-99. Contemp. Math. 338. Amer. Math. Soc., Providence, RI, 2003.

[8] Coulhon, T. And Grigor'yan, A.: On-diagonal lower bounds for heat kernel and Markov chains. Duke Math. J. 89 (1997), 133-199.

[9] Coulhon, T. And Ledoux, M.: Isopérimétrie, décroissance du noyau de la chaleur et transformations de Riesz: un contre-exemple. Ark. Mat. 32 (1994), 63-77.

[10] Coulhon, T. And Saloff-Coste, L.: Variétés riemanniennes isométriques à l'infini. Rev. Mat. Iberoamericana 11 (1995), no. 3, 687-726. 
[11] Coulhon, T. And Saloff-Coste, L.: Isopérimétrie pour les groupes et les variétés. Rev. Mat. Iberoamericana 9 (1993), no. 2, 293-314.

[12] Grigor'yan, A.: Heat kernel upper bounds on a complete non-compact manifold. Rev. Mat. Iberoamericana 10 (1994), no. 2, 395-452.

[13] Gromov, M.: Groups of polynomial growth and expanding maps. Inst. Hautes Études Sci. Publ. Math. 53 (1981), 53-73.

[14] Gromov, M.: Structures métriques pour les variétés riemanniennes. Textes Mathématiques 1. CEDIC, Paris, 1981.

[15] Gromov, M.: Infinite groups as geometric objects. In Proceedings of the International Congress of Mathematicians, Vol. 1, 2 (Warsaw, 1983), 385392. PWN, Warsaw, 1984.

[16] Gromov, M.: Asymptotic invariants of infinite groups. In Geometric group theory, Vol. 2 (Sussex, 1991), 1-295. London Math. Soc. Lecture Note Ser. 182. Cambridge Univ. Press, Cambridge, 1993.

[17] Heinonen, J.: Lectures on analysis on metric spaces. Universitext. Springer-Verlag, New York, 2001.

[18] Hjorth, G.: Classification and orbit equivalence relations. Mathematical Surveys and Monographs 75. American Mathematical Society, Providence, RI, 2000.

[19] Kanai, M.: Rough isometries, and combinatorial approximations of geometries of noncompact Riemannian manifolds. J. Math. Soc. Japan 37 (1985), 391-413.

[20] Kesten, H.: Symmetric random walks on groups. Trans. Amer. Math. Soc. 92 (1959), 336-354.

[21] Pier, J. P.: Amenable locally compact groups. Pure and Applied Mathematics (New York). A Wiley-Interscience Publication. John Wiley \& Sons, Inc., New York, 1984.

[22] Pittet, C.: The isoperimetric profile of homogeneous Riemannian manifolds. J. Differential Geom. 54 (2000), no. 2, 255-302.

[23] Pittet, C. and Saloff-Coste, L.: A survey on the relationships between volume growth, isoperimetry, and the behaviour of simple random walk on Cayley graphs, with examples. Unpublished manuscript, 1997.

[24] Roe, J.: Lectures on coarse geometry. University Lecture Series 31. American Mathematical Society, Providence, RI, 2003.

[25] Saloff-Coste, L.: Analysis on Riemannian co-compact covers. In Surveys in differential Geometry IX, 351-384. International Press, Sommerville, MA, 2004.

[26] Saloff-Coste, L. And Woess, W.: Transition operators on co-compact $G$-spaces. Rev. Mat. Iberoamericana 22 (2006), no. 3, 747-799.

[27] Salvatori, M.: On the norms of group-invariant transition operators on graphs. J. Theoret. Probab. 5 (1992), 563-576. 
[28] Semmes, S.: Finding Curves on General Spaces through quantitative topology, with applications for Sobolev and Poincaré inequalities. Selecta Math. (N.S.) 2 (1996), 155-295.

[29] Soardi, P. M. And Woess, W.: Amenability, unimodularity, and the spectral radius of random walks on infinite graphs. Math. Z. 205 (1990), 471-486.

[30] Tessera, R.: Asymptotic isoperimetry of balls in metric measure spaces. Publ. Math. 50 (2006), no. 2, 315-348.

[31] Tessera, R.: Asymptotic isoperimetry on groups and uniform embeddings into Banach spaces. Preprint, 2006. Available at math.GR/0603138.

[32] Tessera, R.: Vanishing of the first reduced cohomology with values in a $L^{p}$-representation. To appear in Ann. Inst. Fourier (Grenoble).

[33] Varopoulos, N. Th.: Analysis on Lie groups. J. Funct. Anal. 76 (1988), no. $2,346-410$.

Recibido: 16 de marzo de 2007

Revisado: 11 de diciembre de 2007

Romain Tessera

Department of Mathematics

Vanderbilt University

Stevenson Center

Nashville, TN 37240, USA

romain.a.tessera@vanderbilt.edu 